\title{
Shadows of moving surfaces
}

\author{
Wei-Zhi SuN \\ (Received December 12, 1995; Revised February 6, 1996)
}

\begin{abstract}
We classify the bifurcation of generic local pictures of shadows for oneparameter families of surfaces in the Euclidean 3-space.
\end{abstract}

Key words: normal forms of shadow, versal deformation, $t-P-\mathcal{K}$-equivalence.

\section{Introduction}

We consider the problem how the bifurcation of shadows for moving surface looks like. A classification of the shadows of generic submanifolds in $\mathbb{R}^{n}$ was given by Watanabe [12]. In this paper we shall study the normal forms of shadows of one parameter families of surfaces and illustrate how shadows of surfaces change when surfaces move along one parameter in $\mathbb{R}^{3}$.

One of the motivations for the study of the shadows of surfaces is given in Vision Theory $([4],[8])$. In [8], Lions et. al. studied the so-called Shapefrom-Shading problem. This problem corresponds, roughly speaking, to the reconstruction of a shape (a surface) from the brightness of the twodimensional image. They studied this problem as an application of the theory of viscosity solutions for various kinds of boundary value problems for a first order Hamilton-Jacobi equation. The boundary in these problems was considered as the edge of the shadows of a surface.

Let $\mathbb{R}^{3}$ be the Euclidean 3 -space with coordinate $\left(x, y_{1}, y_{2}\right)$. The subset $G$ in $\mathbb{R}^{2}$ is called the shadow of a surface $H$ in $\mathbb{R}^{3}$, if $G$ is the image of projection $\pi$ along a certain direction (for example, $x$-axis), where $\pi: \mathbb{R}^{3} \rightarrow$ $\mathbb{R}^{2}$ is given by $\pi\left(x, y_{1}, y_{2}\right)=\left(y_{1}, y_{2}\right)$.

Let $H$ be a closed surface in $\mathbb{R}^{3}$. We denote the set of embeddings from $H$ to $\mathbb{R}^{3}$ by

$$
\operatorname{Emb}\left(H, \mathbb{R}^{3}\right)=\left\{i: H \hookrightarrow \mathbb{R}^{3} \mid i \text { is an embedding }\right\}
$$

which is a Borel-space if we adopt the Whitney topology. We consider the following set 


$$
\mathcal{P}=\left\{e: H \times I \hookrightarrow \mathbb{R}^{3} \times \mathbb{R} \mid e(p, t)=\left(i_{t}(p), t\right), i_{t} \in \operatorname{Emb}\left(H, \mathbb{R}^{3}\right)\right\},
$$

where $I$ is an open interval in $\mathbb{R}$ which contains the origin. For any $e \in \mathcal{P}, e$ is regarded as a family of elements of $\operatorname{Emb}\left(H, \mathbb{R}^{3}\right)$ with a parameter $t$, and the image $e(H \times I)$ is a 3-dimensional submanifold in $\mathbb{R}^{3} \times \mathbb{R}$.

We suppose that the moving surfaces have the shadow in $\mathbb{R}^{2} \times \mathbb{R}$. For any $e \in \mathcal{P}$, the image of Пoe is called a shadow of $e$, where $\Pi: \mathbb{R}^{3} \times \mathbb{R} \rightarrow \mathbb{R}^{2} \times \mathbb{R}$ is the canonical projection defined by $\Pi\left(x, y_{1}, y_{2}, t\right)=\left(y_{1}, y_{2}, t\right)$. Our purpose in this paper is to give a local classification of the bifurcation of the image of $\Pi \circ e$ along the parameter $t$ under the parameterized diffeomorphisms. The precise definition is given as follows.

Definition 1.1 Let $D$ and $D^{\prime}$ be set germs in $\left(\mathbb{R}^{2} \times \mathbb{R}, 0\right)$. We say that $D$ and $D^{\prime}$ are $t$-diffeomorphic if there exist diffeomorphism germs $\hat{\Phi}:\left(\mathbb{R}^{2} \times\right.$ $\mathbb{R}, 0) \rightarrow\left(\mathbb{R}^{2} \times \mathbb{R}, 0\right)$ and $\hat{\phi}:(\mathbb{R}, 0) \rightarrow(\mathbb{R}, 0)$ such that $\hat{\Phi}(D)=D^{\prime}$ and $\pi_{t} \circ \hat{\Phi}=\hat{\phi} \circ \pi_{t}$, where $\pi_{t}: \mathbb{R}^{2} \times \mathbb{R} \rightarrow \mathbb{R}$ is the projection to the second components.

Under the above notation, we define $D_{t}=D \cap\left(\mathbb{R}^{2} \times\{t\}\right)$ and $D_{t}^{\prime}=$ $D^{\prime} \cap\left(\mathbb{R}^{2} \times\{t\}\right)$. If $D$ and $D^{\prime}$ are $t$-diffeomorphic, then $\hat{\Phi}\left(D_{t}\right)=D_{\hat{\phi}(t)}^{\prime}$, that is the bifurcations of $\left\{D_{t}\right\}_{t \in(\mathbb{R}, 0)}$ and $\left\{D_{t}^{\prime}\right\}_{t \in(\mathbb{R}, 0)}$ along the parameter $t$ are diffeomorphic. Our main result in this paper is the following theorem.

Theorem A There exists a residual subset $\mathcal{Q} \subset \mathcal{P}$ with the following property: For any $e \in \mathcal{Q}$ and for any point $Y_{0}$ of the shadow $\Pi \circ e(H \times I)$, the number $r$ of singular points of $\Pi \circ e$ in $(\Pi \circ e)^{-1}\left(Y_{0}\right)$ is at most 3 and the set germ of the shadow at $Y_{0}$ is t-diffeomorphic to one of the set germ in the following list:

$$
r=1
$$

\begin{tabular}{|c|c|}
\hline${ }^{p} G_{k}$ & normal forms of set germs of the shadows \\
\hline${ }^{0} G_{0}$ & $\left\{\left(y_{1}, y_{2}, t\right) \in \mathbb{R}^{2} \times \mathbb{R} \mid y_{i} \in \mathbb{R}\right\}$ \\
${ }^{0} G_{2}$ & $\left\{\left(y_{1}, y_{2}, t\right) \in \mathbb{R}^{2} \times \mathbb{R} \mid y_{1} \leqslant 0\right\}$ \\
\hline${ }^{0} G_{1}$ & $\left\{\left(y_{1}, y_{2}, t\right) \in \mathbb{R}^{2} \times \mathbb{R} \mid y_{i} \in \mathbb{R}\right\}$ \\
\hline${ }^{1} G_{2}^{+}$ & \\
${ }^{1} G_{2}^{-}$ & $\left\{\left(y_{1}, y_{2}, t\right) \in \mathbb{R}^{2} \times \mathbb{R} \mid 27 y_{2}^{4}-256 y_{1}^{3}-144 y_{1} y_{2}^{2} t\right.$ \\
\hline${ }^{1} G_{3}$ & $\left.+4 y_{2}^{2} t^{3}-16 y_{1} t^{4}+128 y_{1}^{2} t^{2} \leqslant 0\right\}$ \\
& \\
\hline
\end{tabular}


$r=2$

\begin{tabular}{|c|c|}
\hline${ }^{p} G_{k}$ & normal forms of set germs of 2-multi- shadows \\
\hline${ }^{0} G_{1,1}$ & $\left\{\left(y_{1}, y_{2}, t\right) \in \mathbb{R}^{2} \times \mathbb{R} \mid y_{1} \leqslant 0\right\}$ \\
& $\cup\left\{\left(y_{1}, y_{2}, t\right) \in \mathbb{R}^{2} \times \mathbb{R} \mid y_{2} \leqslant 0\right\}$ \\
\hline${ }^{1} G_{1,1}^{+}$ & $\left\{\left(y_{1}, y_{2}, t\right) \in \mathbb{R}^{2} \times \mathbb{R} \mid y_{2}^{2}+t+y_{1} \leqslant 0\right\}$ \\
& $\cup\left\{\left(y_{1}, y_{2}, t\right) \in \mathbb{R}^{2} \times \mathbb{R} \mid y_{1} \leqslant 0\right\}$ \\
\hline${ }^{1} G_{1,1}^{-}$ & $\left\{\left(y_{1}, y_{2}, t\right) \in \mathbb{R}^{2} \times \mathbb{R} \mid y_{2}^{2}-t+y_{1} \leqslant 0\right\}$ \\
& $\cup\left\{\left(y_{1}, y_{2}, t\right) \in \mathbb{R}^{2} \times \mathbb{R} \mid y_{1} \leqslant 0\right\}$ \\
\hline${ }^{1} G_{2,1}^{+}$ & $\left\{\left(y_{1}, y_{2}, t\right) \in \mathbb{R}^{2} \times \mathbb{R} \mid y_{i} \in \mathbb{R}\right\}$ \\
${ }^{1} G_{2,1}^{-}$ & $\cup\left\{\left(y_{1}, y_{2}, t\right) \in \mathbb{R}^{2} \times \mathbb{R} \mid y_{2} \leqslant 0\right\}$ \\
\hline${ }^{1} G_{1,2}^{+}$ & $\left\{\left(y_{1}, y_{2}, t\right) \in \mathbb{R}^{2} \times \mathbb{R} \mid y_{2}+t \leqslant 0\right\}$ \\
${ }^{1} G_{1,2}^{-}$ & $\cup\left\{\left(y_{1}, y_{2}, t\right) \in \mathbb{R}^{2} \times \mathbb{R} \mid y_{i} \in \mathbb{R}\right\}$ \\
\hline
\end{tabular}

$r=3$

\begin{tabular}{|c|c|}
\hline${ }^{p} G_{k}$ & normal forms of set germs of 3 -multi- shadows \\
\hline${ }^{1} G_{1,1,1}^{+}$ & $\left\{\left(y_{1}, y_{2}, t\right) \in \mathbb{R}^{2} \times \mathbb{R} \mid y_{1}+y_{2}+t \leqslant 0\right\}$ \\
& $\cup\left\{\left(y_{1}, y_{2}, t\right) \in \mathbb{R}^{2} \times \mathbb{R} \mid y_{1} \leqslant 0\right\}$ \\
& $\cup\left\{\left(y_{1}, y_{2}, t\right) \in \mathbb{R}^{2} \times \mathbb{R} \mid y_{2} \leqslant 0\right\}$ \\
\hline${ }^{1} G_{1,1,1}^{-}$ & $\left\{\left(y_{1}, y_{2}, t\right) \in \mathbb{R}^{2} \times \mathbb{R} \mid y_{1}+y_{2}-t \leqslant 0\right\}$ \\
& $\cup\left\{\left(y_{1}, y_{2}, t\right) \in \mathbb{R}^{2} \times \mathbb{R} \mid y_{1} \leqslant 0\right\}$ \\
& $\cup\left\{\left(y_{1}, y_{2}, t\right) \in \mathbb{R}^{2} \times \mathbb{R} \mid y_{2} \leqslant 0\right\}$ \\
\hline
\end{tabular}

The situation is depicted as follows :

$r=1$

${ }^{0} G_{0}$

${ }^{0} G_{2}$
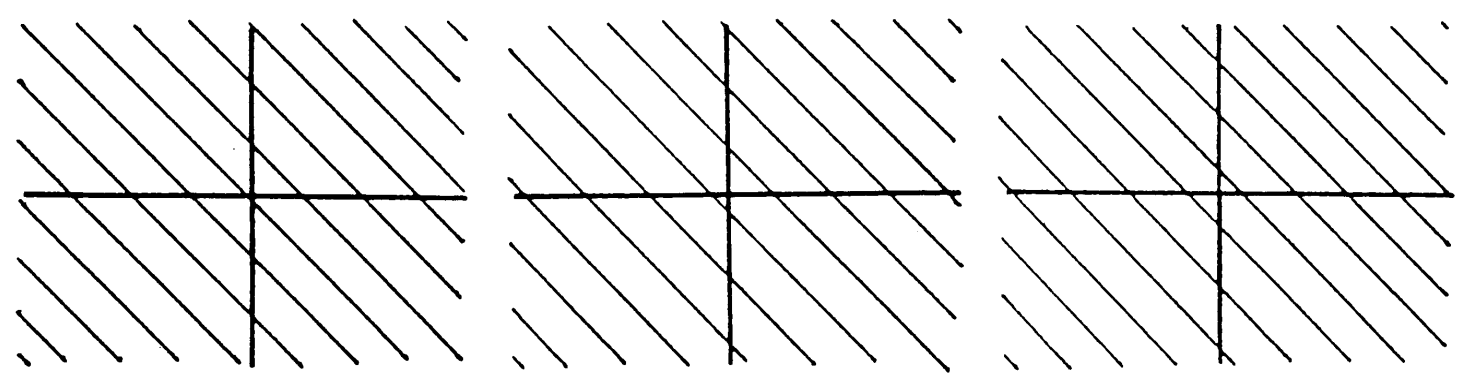
${ }^{0} G_{1}$
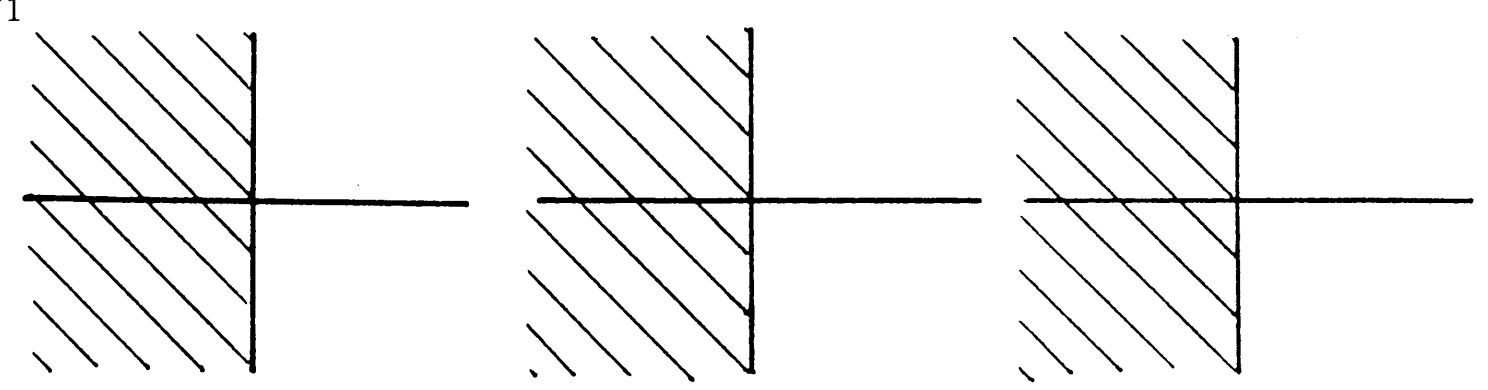

${ }^{1} G_{2}^{+}$
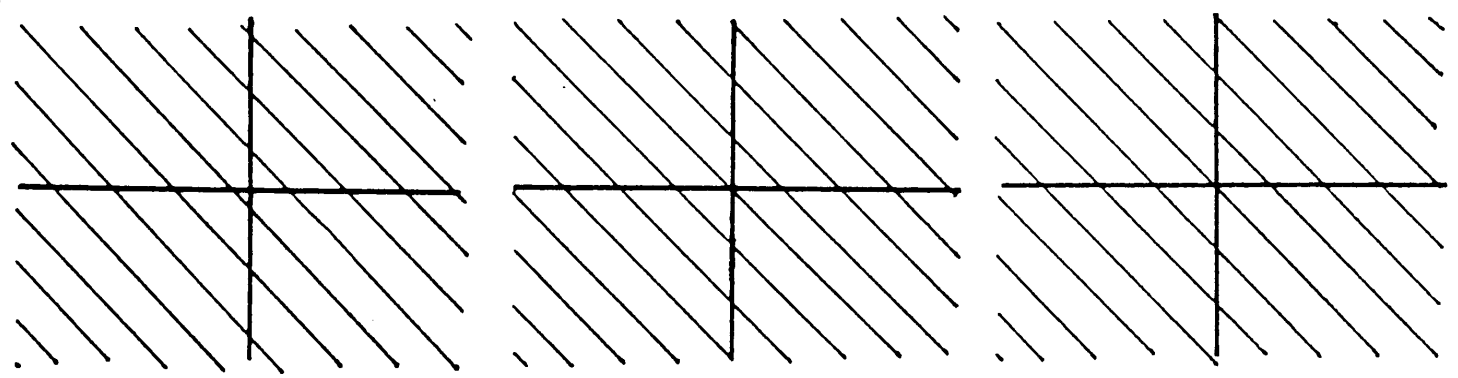
${ }^{1} G_{3}$
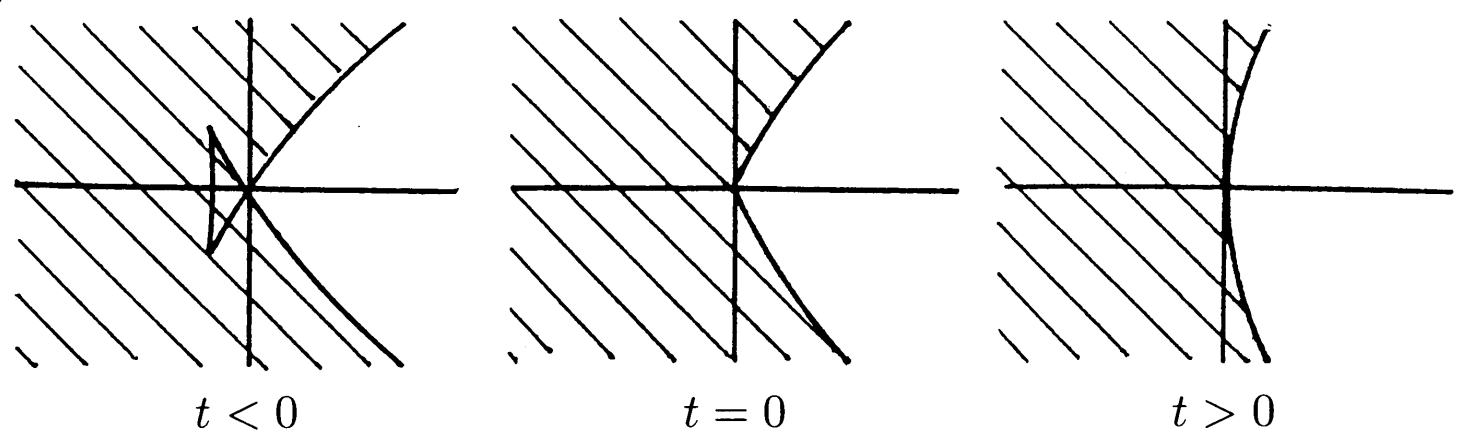

$$
r=2
$$

${ }^{0} G_{1,1}$
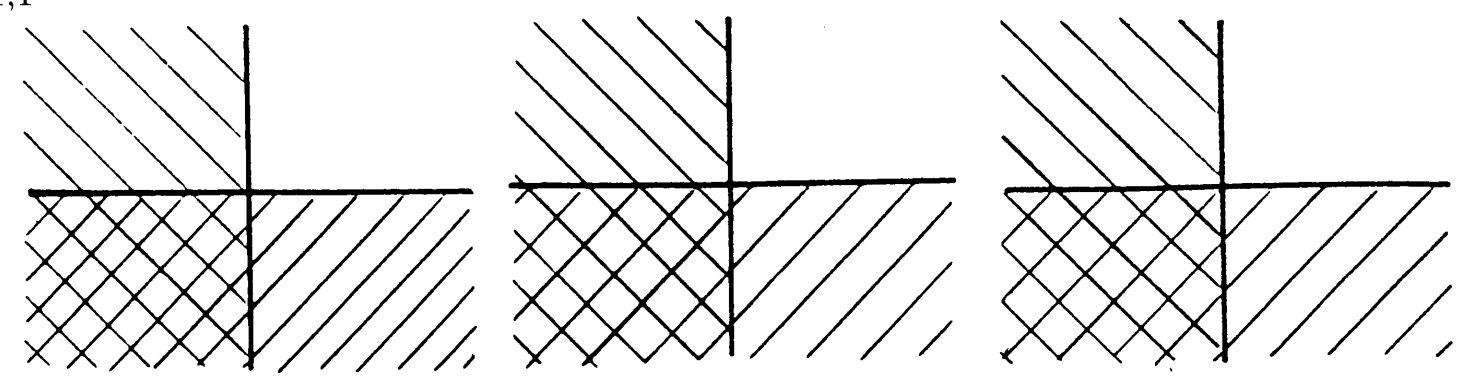

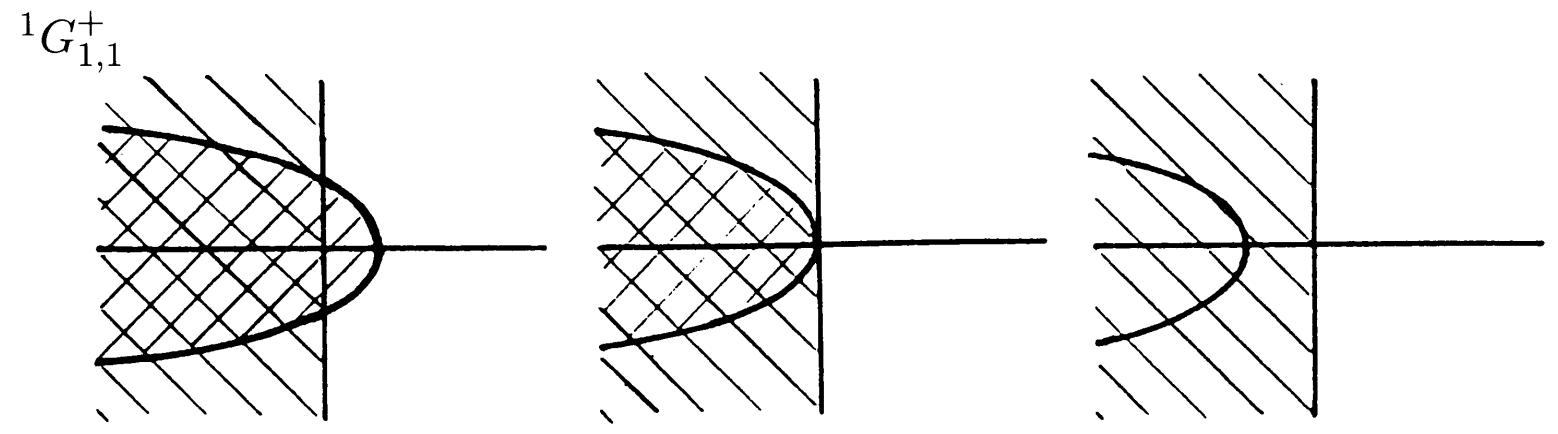

${ }^{1} G_{1,1}^{-}$
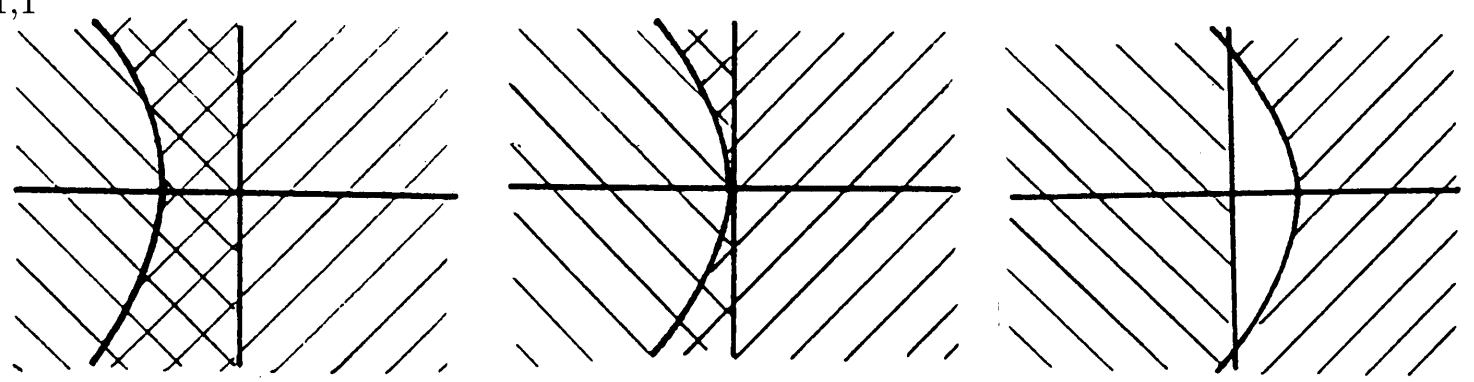
${ }^{1} G_{2,1}^{+} ;{ }^{1} G_{2,1}^{-}$
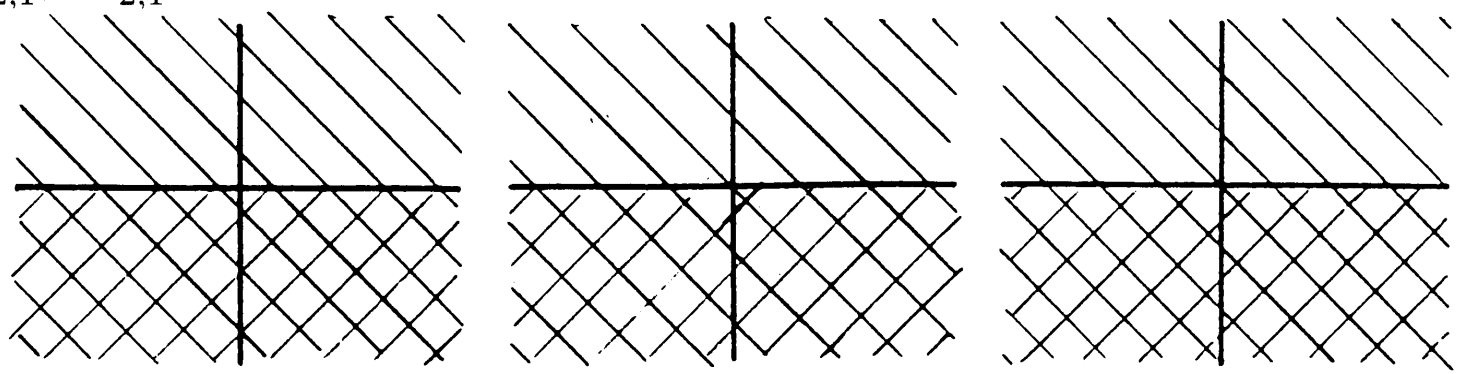
${ }^{1} G_{1,2}^{+} ;{ }^{1} G_{1,2}^{-}$
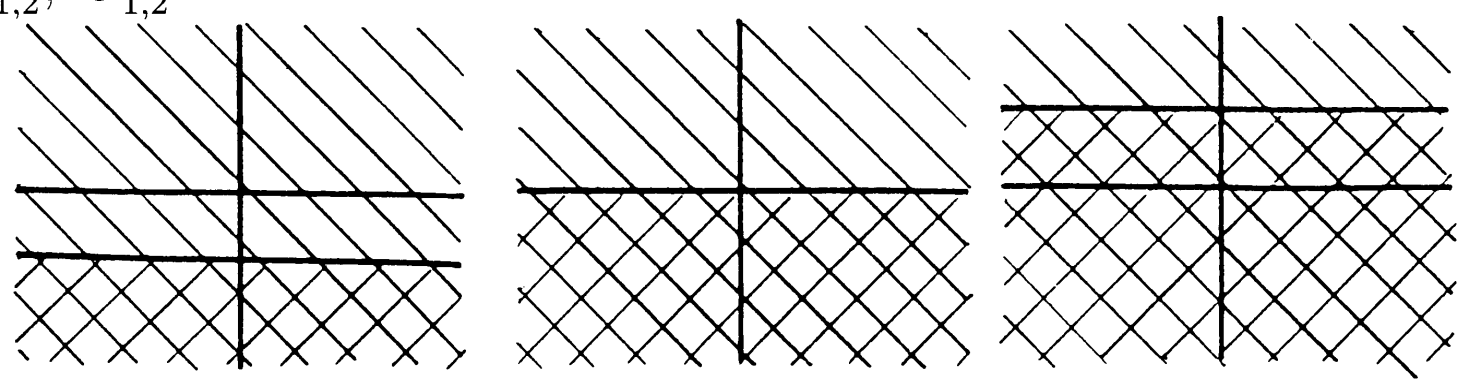

$r=3$

$$
t<0
$$

$$
t=0
$$

$t>0$
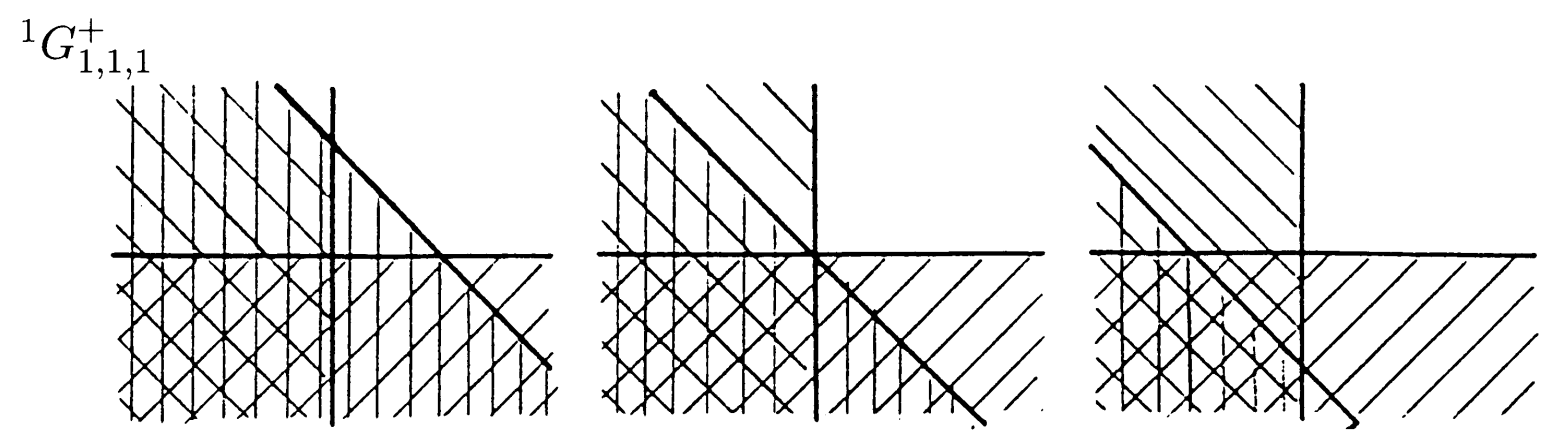


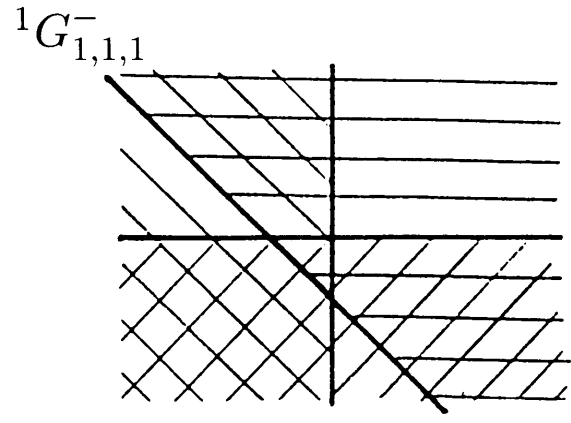

$t<0$

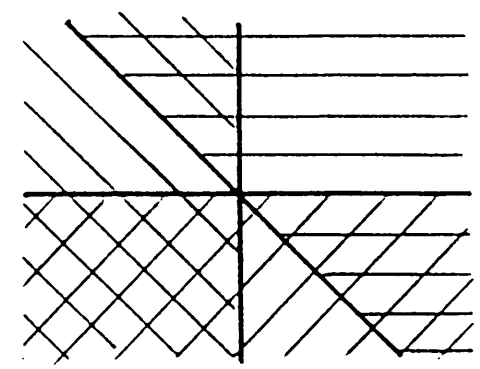

$t=0$

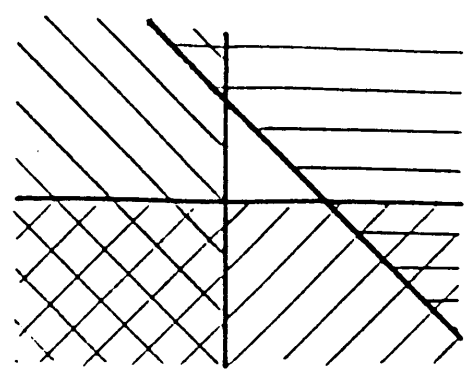

$t>0$

The above classification of shadows is obtained via a classification of defining functions of embedded surfaces $e(H \times I)$. (See Theorem 2.3 and Theorem 4.4. See also Proposition 2.2). The notation ${ }^{p} G_{k}^{( \pm)}$for the normal forms of shadows is named after the notation ${ }^{p} A_{k}^{( \pm)}$for the normal forms of the defining functions. Therefore Theorem A gives information about not only the shadows but also the locations of the embedded surfaces $e(H \times I)$ from which the shadows come.

The Theorem A is divided into Theorem 3.1 in $\S 3$ and Theorem 4.9 in $\S 4$. In Theorem 3.1 we consider the case the number $r$ of singular points of $\Pi \circ e$ in $(\Pi \circ e)^{-1}\left(Y_{0}\right)$ is 1 . In Theorem 4.9, we consider the other case $r=2$ and 3. The proof of Theorem 4.9 is almost the same as that of Theorem 3.1 , so that we omit the details. The idea of the proof of Theorem 3.1 is summarized as follows : Since the image of $e$ is a hypersurface in $\mathbb{R} \times \mathbb{R}^{2} \times \mathbb{R}$, it may be locally considered as a zero point set of a submersion $F:(\mathbb{R} \times$ $\left.\mathbb{R}^{2} \times \mathbb{R}, 0\right) \rightarrow(\mathbb{R}, 0)$. We apply Zakalyukin's classifications $([13])$ among such function germs up to a certain equivalence relation, which preserves the bifurcation of shadows. We can translate such a classification into the classification of $\Pi_{F}:\left(F^{-1}(0), 0\right) \rightarrow\left(\mathbb{R}^{2} \times \mathbb{R}, 0\right)$ which corresponds to the local classification of $\Pi \circ e$ around a point. After that we apply the Thom's transversality theorem to detect the generic condition on $e$. We use the multi-germ version of the above arguments to prove Theorem 4.9.

In $\S 2$, we study the local properties of submanifold $e(H \times I)$ around a single point. In $\S 3$, we give a proof of Theorem 3.1. In $\S 4$, we study the case $r=2,3$ and give a proof of Theorem 4.9.

All map germs considered here are differentiable of class $C^{\infty}$, unless stated otherwise. 


\section{Classification of the local shadows}

In this section we prepare some local theory for the study of shadows.

Let $e \in \mathcal{P}$. For any $\left(p_{0}, t_{0}\right) \in H \times I$, since $e(H \times I)$ is a 3 -dimensional submanifold in $\mathbb{R} \times \mathbb{R}^{2} \times \mathbb{R}$, it follows from the implicit function theorem that there exists a small neighborhood $U$ of $e\left(p_{0}, t_{0}\right)$ in $\mathbb{R} \times \mathbb{R}^{2} \times \mathbb{R}$ and a function $F: U \rightarrow \mathbb{R}$ such that $\left.F\right|_{U \cap \mathbb{R} \times \mathbb{R}^{2} \times\left\{t_{0}\right\}}$ is a submersion and

$$
F^{-1}(0)=U \cap e(H \times I) .
$$

We call $F$ a local equation of $e$ at $e\left(p_{0}, t_{0}\right)$.

Since we consider the local theory, It suffices to study submersion $F$ : $\left(\mathbb{R} \times \mathbb{R}^{2} \times \mathbb{R}, 0\right) \rightarrow(\mathbb{R}, 0)$ at the origin.

Definition 2.1 Let $F, F^{\prime}:\left(\mathbb{R} \times \mathbb{R}^{2} \times \mathbb{R}, 0\right) \rightarrow(\mathbb{R}, 0)$ be function germs. We say that $F$ and $F^{\prime}$ are $t-(P-\mathcal{K})$-equivalent if there exists a diffeomorphism germ

$$
\Phi:\left(\mathbb{R} \times \mathbb{R}^{2} \times \mathbb{R}, 0\right) \rightarrow\left(\mathbb{R} \times \mathbb{R}^{2} \times \mathbb{R}, 0\right)
$$

of the form

$$
\Phi\left(x, y_{1}, y_{2}, t\right)=\left(\phi_{1}\left(x, y_{1}, y_{2}, t\right), \phi_{2}\left(y_{1}, y_{2}, t\right), \phi_{3}(t)\right)
$$

such that

$$
\Phi^{*}<F>_{\mathcal{E}_{\left(x, y_{1}, y_{2}, t\right)}}=<F^{\prime}>_{\mathcal{E}_{\left(x, y_{1}, y_{2}, t\right)}},
$$

where $\mathcal{E}_{\left(\S, \dagger_{\infty}, \dagger_{\epsilon}, \sqcup\right)}$ denotes the ring consisting of function germs $\left(\mathbb{R} \times \mathbb{R}^{2} \times\right.$ $\mathbb{R}, 0) \rightarrow(\mathbb{R}, 0)$.

We remark that the following diagram commutes :

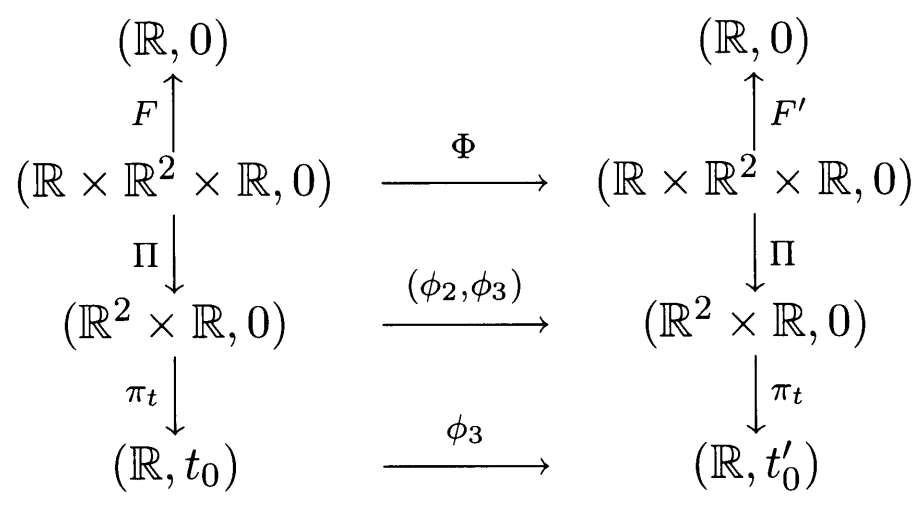

It is clear that $\left(\phi_{2}, \phi_{3}\right):\left(\mathbb{R}^{2} \times \mathbb{R}, 0\right) \rightarrow\left(\mathbb{R}^{2} \times \mathbb{R}, 0\right)$ and $\phi_{3}:(\mathbb{R}, 0) \rightarrow$ 
$(\mathbb{R}, 0)$ are the diffeomorphisms.

Similarly we may define the $t-(P-\mathcal{K})$-equivalence for function germs at arbitrary base points. We have the following proposition.

Proposition 2.2 Let $F, F^{\prime}:\left(\mathbb{R} \times \mathbb{R}^{2} \times \mathbb{R}, 0\right) \rightarrow(\mathbb{R}, 0)$ be function germs. If $F, F^{\prime}$ are $t-(P-\mathcal{K})$-equivalent then $\Pi\left(F^{-1}(0)\right)$ and $\Pi\left(F^{\prime-1}(0)\right)$ are $t$ diffeomorphic.

Proof. By definition, there exists a diffeomorphism germ $\Phi=\left(\phi_{1}, \phi_{2}, \phi_{3}\right)$, such that

$$
<F^{\prime} \circ \Phi>_{\mathcal{E}_{\left(x, y_{1}, y_{2}, t\right)}}=<F>_{\mathcal{E}_{\left(x, y_{1}, y_{2}, t\right)}},
$$

so that $F^{-1}(0)=\Phi^{-1}\left(F^{\prime-1}(0)\right)$. By the commutative diagram, we obtain

$$
\left(\phi_{2}, \phi_{3}\right)\left(\Pi\left(F^{-1}(0)\right)\right)=\Pi\left(F^{\prime-1}(0)\right) .
$$

Set $\hat{\Phi}=\left(\phi_{2}, \phi_{3}\right)$ and $\hat{\phi}=\phi_{3}$, then we have $\hat{\Phi}\left(\Pi\left(F^{-1}(0)\right)\right)=\Pi\left(F^{\prime-1}(0)\right)$ and $\pi_{t} \circ \hat{\Phi}=\hat{\phi} \circ \pi_{t}$, where $\pi_{t}: \mathbb{R}^{2} \times \mathbb{R} \rightarrow \mathbb{R}$ is the projection to the second component.

For the local case, by Proposition 2.2, it is sufficient to consider the local shadows of local equations $F$, that is, the image of $\Pi_{F}=\left.\Pi\right|_{F^{-1}(0)}$ : $\left(F^{-1}(0), 0\right) \rightarrow\left(\mathbb{R}^{2} \times \mathbb{R}, 0\right)$. For $f=\left.F\right|_{\mathbb{R} \times \mathbb{R}^{2} \times\{0\}}$, we consider the subspaces of $\mathcal{E}_{\left(x, y_{1}, y_{2}\right)}$ given by

$$
T_{e}(P-\mathcal{K})(f)=\left\langle\frac{\partial f}{\partial x}, f\right\rangle_{\mathcal{E}_{\left(x, y_{1}, y_{2}\right)}}+\left\langle\frac{\partial f}{\partial y_{1}}, \frac{\partial f}{\partial y_{2}}\right\rangle_{\mathcal{E}_{\left(y_{1}, y_{2}\right)}} .
$$

We also consider its codimensions

$$
(P-\mathcal{K})_{e}-\operatorname{cod}(f)=\operatorname{dim}_{\mathbb{R}} \mathcal{E}_{\left(x, y_{1}, y_{2}\right)} / T_{e}(P-\mathcal{K})(f) .
$$

Let $F:\left(\mathbb{R} \times \mathbb{R}^{2} \times \mathbb{R}, 0\right) \rightarrow(\mathbb{R}, 0)$ be a function germ, we say that $F$ is a $(P-\mathcal{K})$-versal deformation of $f=\left.F\right|_{\mathbb{R} \times \mathbb{R}^{2} \times\{0\}}:\left(\mathbb{R} \times \mathbb{R}^{2} \times\{0\}, 0\right) \rightarrow(\mathbb{R}, 0)$ if

$$
\left\langle\left.\frac{\partial F}{\partial t}\right|_{t=0}\right\rangle_{\mathbb{R}}+T(P-\mathcal{K})_{e}(f)=\mathcal{E}_{\left(x, y_{1}, y_{2}\right)} .
$$

In [7], Zakalyukin's classification theorem is developed to the following theorem which is useful for classification of local equations.

Theorem 2.3 Let $F:\left(\mathbb{R} \times \mathbb{R}^{n} \times \mathbb{R}, 0\right) \rightarrow(\mathbb{R}, 0)$ be a function germ with 
$(P-\mathcal{K})_{e}-\operatorname{cod}(f) \leqslant 1$, where $f=\left.F\right|_{\mathbb{R} \times \mathbb{R}^{n} \times\{0\}}$. If $F$ is $(P-\mathcal{K})$-versal deformation of $f$, then $F$ is $t-(P-\mathcal{K})$-equivalent to one of the germs in the following list :

$$
\begin{array}{ccr}
{ }^{0} A_{k}: & x^{k+1}+\sum_{i=1}^{k} y_{i} x^{i-1} & (0 \leqslant k \leqslant n) \\
{ }^{1} A_{k}: & x^{k+1}+x^{k-1}\left(t \pm y_{k}^{2} \pm \cdots \pm y_{n}^{2}\right)+\sum_{i=1}^{k-1} y_{i} x^{i-1} & (2 \leqslant k \leqslant n+1)
\end{array}
$$

In the case $n=2$, by Theorem 2.3, we have the following corollary.

Corollary 2.4 Let $F:\left(\mathbb{R} \times \mathbb{R}^{2} \times \mathbb{R}, 0\right) \rightarrow(\mathbb{R}, 0)$ be a function germ with $(P-\mathcal{K})_{e}-\operatorname{cod}(f) \leqslant 1$, where $f=\left.F\right|_{\mathbb{R} \times \mathbb{R}^{2} \times\{0\}}$. If $F$ is a $(P-\mathcal{K})$-versal deformation of $f$, then $F$ is $t-(P-\mathcal{K})$-equivalent to one of the following function germs :

$$
\begin{aligned}
& { }^{0} A_{0}: x \\
& { }^{0} A_{1}: x^{2}+y_{1} \\
& { }^{0} A_{2}: x^{3}+x y_{2}+y_{1} \\
& { }^{1} A_{2}^{+}: x^{3}+x y_{2}^{2}+t x+y_{1} \\
& { }^{1} A_{2}^{-}: x^{3}-x y_{2}^{2}+t x+y_{1} \\
& { }^{1} A_{3}: x^{4}+x y_{2}+t x^{2}+y_{1} .
\end{aligned}
$$

We denote the shadow of ${ }^{p} A_{k}^{( \pm)}$by ${ }^{p} G_{k}^{( \pm)}$. Then by Theorem 2.3 we also have the following corollary.

Corollary 2.5 Let $F:\left(\mathbb{R} \times \mathbb{R}^{2} \times \mathbb{R}, 0\right) \rightarrow(\mathbb{R}, 0)$ be a function germ with $(P-\mathcal{K})_{e}-\operatorname{cod}(f) \leqslant 1$, where $f=\left.F\right|_{\mathbb{R} \times \mathbb{R}^{2} \times\{0\}}$. If $F$ is a $(P-\mathcal{K})$-versal deformation of $f$, then $\Pi\left(F^{-1}(0)\right)$ is t-diffeomorphism to one of the set germs in the above list ${ }^{p} G_{k}^{( \pm)}$(See the following table).

\begin{tabular}{|c|c|}
\hline${ }^{p} G_{k}$ & normal forms of set germs of the shadows \\
\hline${ }^{0} G_{0}$ & $\left\{\left(y_{1}, y_{2}, t\right) \in \mathbb{R}^{2} \times \mathbb{R} \mid y_{i} \in \mathbb{R}\right\}$ \\
\hline${ }^{0} G_{1}$ & $\left\{\left(y_{1}, y_{2}, t\right) \in \mathbb{R}^{2} \times \mathbb{R} \mid y_{1} \leqslant 0\right\}$ \\
\hline${ }^{0} G_{2}$ & $\left\{\left(y_{1}, y_{2}, t\right) \in \mathbb{R}^{2} \times \mathbb{R} \mid y_{i} \in \mathbb{R}\right\}$ \\
\hline${ }^{1} G_{2}^{+}$ & $\left\{\left(y_{1}, y_{2}, t\right) \in \mathbb{R}^{2} \times \mathbb{R} \mid y_{i} \in \mathbb{R}\right\}$ \\
\hline${ }^{1} G_{2}^{-}$ & $\left\{\left(y_{1}, y_{2}, t\right) \in \mathbb{R}^{2} \times \mathbb{R} \mid y_{i} \in \mathbb{R}\right\}$ \\
\hline${ }^{1} G_{3}$ & $\left\{\left(y_{1}, y_{2}, t\right) \in \mathbb{R}^{2} \times \mathbb{R} \mid 27 y_{2}^{4}-256 y_{1}^{3}-144 y_{1} y_{2}^{2} t\right.$ \\
& $\left.+4 y_{2}^{2} t^{3}-16 y_{1} t^{4}+128 y_{1}^{2} t^{2} \leqslant 0\right\}$ \\
\hline
\end{tabular}


Remark. When $p=1$ and $k=3$, we observe that $x^{4}+t x^{2}+x y_{2}+y_{1}$ is $t-(P-\mathcal{K})$-equivalent to $x^{4}-t x^{2}+x y_{2}+y_{1}$.

In order to study the generic properties of $e \in \mathcal{P}$ which respect to the local equation $F$ at $e\left(p_{0}, t_{0}\right)$, we need some preparations.

Let $g:\left(\mathbb{R}^{2}, 0\right) \rightarrow\left(\mathbb{R}^{2}, 0\right)$ be a $C^{\infty}$ germ. In [2], two types of codimensions of $g$ are defined as follows :

$$
(\mathcal{A})-\operatorname{cod}(g)=\operatorname{dim}_{\mathbb{R}} \mathfrak{M}_{2} \times \mathfrak{M}_{2} / T(\mathcal{A})(g)
$$

and

$$
(\mathcal{A})_{e}-\operatorname{cod}(g)=\operatorname{dim}_{\mathbb{R}} \mathcal{E}_{2} \times \mathcal{E}_{2} / T_{e}(\mathcal{A})(g)
$$

where

$$
T(\mathcal{A})(g)=\mathfrak{M}_{2}\left\langle\frac{\partial g}{\partial x_{1}}, \frac{\partial g}{\partial x_{2}}\right\rangle_{\mathcal{E}_{2}}+g^{*} \mathfrak{M}_{2} \times g^{*} \mathfrak{M}_{2}
$$

and

$$
T_{e}(\mathcal{A})(g)=\left\langle\frac{\partial g}{\partial x_{1}}, \frac{\partial g}{\partial x_{2}}\right\rangle_{\mathcal{E}_{2}}+g^{*} \mathcal{E}_{2} \times g^{*} \mathcal{E}_{2}
$$

Remark. $T(\mathcal{A})(g)$ and $T(\mathcal{A})_{e}(g)$ do not depend on the choice of the local coordinates on the source and the target.

In $([1],[6])$, the notion of the versality for deformations is defined as follows.

Let $G:\left(\mathbb{R}^{2} \times \mathbb{R}, 0\right) \rightarrow\left(\mathbb{R}^{2}, 0\right)$ be a $C^{\infty}$-map germ and $g=\left.G\right|_{\mathbb{R}^{2} \times\{0\}}$ : $\left(\mathbb{R}^{2}, 0\right) \rightarrow\left(\mathbb{R}^{2}, 0\right)$. We say that $G$ is an $\mathcal{A}$-versal deformation of $g$ if

$$
\left\langle\left.\frac{\partial G}{\partial t}\right|_{t=0}\right\rangle_{\mathbb{R}}+T(\mathcal{A})_{e}(g)=\mathcal{E}_{2} \times \mathcal{E}_{2}
$$

We now consider a map germ

$$
j_{1}^{\ell} G:\left(\mathbb{R}^{2} \times \mathbb{R}, 0\right) \rightarrow J^{\ell}\left(\mathbb{R}^{2}, \mathbb{R}^{2}\right) \cong \mathbb{R}^{2} \times \mathbb{R}^{2} \times J^{\ell}(2,2)
$$

given by

$$
j_{1}^{\ell} G(x, t)=j^{\ell} G_{t}(x) .
$$

Let $z=j^{\ell} g(0)$ and $L^{\ell}(2) \times L^{\ell}(2)(z)$ be the $\mathcal{A}$-orbit through $z$ in $J^{\ell}(2,2)$ (See [2], [5]). 
Lemma 2.6 Suppose that $g=\left.G\right|_{t=0}$ is $\mathcal{A}$-finitely determined $\left(\right.$ i.e. $(\mathcal{A})_{e}-$ $\operatorname{cod}(g)<+\infty)$. Under the above notations, for sufficiently large $\ell$, the following conditions are equivalent.

(i) $\tilde{\pi} \circ j_{1}^{\ell} G \bar{\pitchfork}\left(L^{\ell}(2) \times L^{\ell}(2)\right)(z)$.

(ii) $G$ is an $\mathcal{A}$-versal deformation of $g$,

where $\tilde{\pi}: \mathbb{R}^{2} \times \mathbb{R}^{2} \times J^{\ell}(2,2) \rightarrow J^{\ell}(2,2)$ is the canonical projection.

Proof. By the definition of the transversality,

$$
\tilde{\pi} \circ j_{1}^{\ell} G \bar{\pitchfork}\left(L^{\ell}(2) \times L^{\ell}(2)\right)(z)
$$

if and only if

$$
\begin{aligned}
d\left(\tilde{\pi} \circ j_{1}^{\ell} G\right)_{(0,0)}\left(T_{(0,0)} \mathbb{R}^{2} \times \mathbb{R}\right) & +T_{z}\left(L^{\ell}(2) \times L^{\ell}(2)\right)(z) \\
& =T_{z} J^{\ell}(2,2) .
\end{aligned}
$$

It is also equivalent to the following condition :

$$
\begin{aligned}
\pi_{\ell}^{-1}\left(d\left(\tilde{\pi} \circ j_{1}^{\ell} G\right)_{(0,0)}\left(T_{(0,0)} \mathbb{R}^{2} \times \mathbb{R}\right)\right) & +T(\mathcal{A})(g)+\mathfrak{M}_{2}^{\ell+1} \times \mathfrak{M}_{2}^{\ell+1} \\
& =\mathfrak{M}_{2} \times \mathfrak{M}_{2},
\end{aligned}
$$

where $\pi_{\ell}: \mathfrak{M}_{2} \times \mathfrak{M}_{2} \rightarrow J^{\ell}(2,2)$ is the canonical projection. Therefore we have

$$
\begin{aligned}
& \pi_{\ell}^{-1}\left(d\left(\tilde{\pi} \circ j_{1}^{\ell} G\right)_{(0,0)}\left(T_{(0,0)} \mathbb{R}^{2} \times \mathbb{R}\right)\right) \\
& =\left\langle\pi_{\ell}^{-1}\left(d\left(\tilde{\pi} \circ j_{1}^{\ell} G\right)_{(0,0)}\left(\frac{\partial}{\partial x_{1}}\right)\right), \pi_{\ell}^{-1}\left(d\left(\tilde{\pi} \circ j_{1}^{\ell} G\right)_{(0,0)}\left(\frac{\partial}{\partial x_{2}}\right)\right)\right. \\
& \left.\quad \pi_{\ell}^{-1}\left(d\left(\tilde{\pi} \circ j_{1}^{\ell} G\right)_{(0,0)}\left(\frac{\partial}{\partial t}\right)\right)\right\rangle_{\mathbb{R}} \\
& \quad+\mathfrak{M}_{2}^{\ell+1} \times \mathfrak{M}_{2}^{\ell+1} .
\end{aligned}
$$

Then (1) is equivalent to

$$
\left\langle\frac{\partial g}{\partial x_{1}}, \frac{\partial g}{\partial x_{2}},\left.\frac{\partial G}{\partial t}\right|_{t=0}\right\rangle_{\mathbb{R}}+T(\mathcal{A})(g)+\mathfrak{M}_{2}^{\ell+1} \times \mathfrak{M}_{2}^{\ell+1}=\mathfrak{M}_{2} \times \mathfrak{M}_{2}
$$

It follows from the definitions of $T(\mathcal{A})(g)$ and $T(\mathcal{A})_{e}(g)$ that $(2)$ is equivalent to

$$
\left\langle\left.\frac{\partial G}{\partial t}\right|_{t=0}\right\rangle_{\mathbb{R}}+T(\mathcal{A})_{e}(g)+\mathfrak{M}_{2}^{\ell+1} \times \mathfrak{M}_{2}^{\ell+1}=\mathcal{E}_{2} \times \mathcal{E}_{2}
$$


Since $g$ is $\mathcal{A}$-finitely determined, we have

$$
\mathfrak{M}_{2}^{\ell+1} \times \mathfrak{M}_{2}^{\ell+1} \subset T(\mathcal{A})(g) \quad \text { for some } \ell .
$$

Then (3) is equivalent to

$$
\left\langle\left.\frac{\partial G}{\partial t}\right|_{t=0}\right\rangle_{\mathbb{R}}+T(\mathcal{A})_{e}(g)=\mathcal{E}_{2} \times \mathcal{E}_{2}
$$

Let $F:\left(\mathbb{R} \times \mathbb{R}^{2} \times \mathbb{R}, 0\right) \rightarrow(\mathbb{R}, 0)$ be a function germ such that $f=$ $\left.F\right|_{\mathbb{R} \times \mathbb{R}^{2} \times\{0\}}:\left(\mathbb{R} \times \mathbb{R}^{2} \times\{0\}, 0\right) \rightarrow(\mathbb{R}, 0)$ is a submersion germ. We consider the local projection $\Pi_{F}=\left.\Pi\right|_{F^{-1}(0)}:\left(F^{-1}(0), 0\right) \rightarrow\left(\mathbb{R}^{2} \times \mathbb{R}, 0\right)$. and $\pi_{f}=$ $\left.\pi\right|_{f^{-1}(0) \times\{0\}}:\left(f^{-1}(0), 0\right) \rightarrow\left(\mathbb{R}^{2} \times\{0\}, 0\right)$.

By the above remark, $T(\mathcal{A})\left(\pi_{f}\right)$ and $T(\mathcal{A})_{e}\left(\pi_{f}\right)$ are well-defined. Therefore $\mathcal{A}$-versality of deformation $\Pi_{F}$ of $\pi_{f}$ is also well-defined.

Under the above notations, we have the following proposition.

Proposition 2.3 The following conditions are equivalent.

(i) $F$ is a $(P-\mathcal{K})$-versal deformation of $f$.

(ii) $\pi_{2} \circ \Pi_{F}$ is an $\mathcal{A}$-versal deformation of $\pi_{f}$.

Here $\pi_{2}:\left(\mathbb{R}^{2} \times \mathbb{R}, 0\right) \rightarrow\left(\mathbb{R}^{2}, 0\right)$ is the canonical projection.

Proof. Since $f$ is a submersion, we may suppose that $\frac{\partial F}{\partial y_{1}} \neq 0$ (for the case $\frac{\partial F}{\partial x} \neq 0$ or $\frac{\partial F}{\partial y_{2}} \neq 0$ are similar), then we may suppose that $F$ has the form $F\left(x, y_{1}, y_{2}, t\right)=y_{1}-h\left(x, y_{2}, t\right)$, for some function $h:(\mathbb{R} \times \mathbb{R} \times$ $\mathbb{R}, 0) \rightarrow(\mathbb{R}, 0)$ and $f\left(x, y_{1}, y_{2}\right)=F\left(x, y_{1}, y_{2}, 0\right)=y_{1}-h_{0}\left(x, y_{2}\right)$, where $h_{0}\left(x, y_{2}\right)=h\left(x, y_{2}, 0\right)$. Define $G_{F}:\left(\mathbb{R}^{2} \times \mathbb{R}, 0\right) \rightarrow\left(\mathbb{R}^{2}, 0\right)$ by $G_{F}\left(x, y_{2}, t\right)=$ $\left(h\left(x, y_{2}, t\right), y_{2}\right)$ and $g_{f}\left(x, y_{2}\right)=\left(h_{0}\left(x, y_{2}\right), y_{2}\right)$. Then $G_{F}=\pi_{2} \circ \Pi_{F}$ and $g_{f}=\pi_{f}$. We consider the map germ $I_{h_{0}}:\left(\mathbb{R}^{2}, 0\right) \rightarrow\left(\mathbb{R}^{3}, 0\right)$ defined by

$$
I_{h_{0}}\left(x, y_{2}\right)=\left(x, h_{0}\left(x, y_{2}\right), y_{2}\right)
$$

and we also consider the pull-back homomorphism

$$
I_{h_{0}}^{*}: \mathcal{E}_{\left(x, y_{1}, y_{2}\right)} \rightarrow \mathcal{E}_{\left(x, y_{2}\right)} .
$$

Then $\operatorname{ker}_{h_{0}}^{*}=\left\langle y_{1}-h_{0}\left(x, y_{2}\right)\right\rangle_{\mathcal{E}_{\left(x, y_{1}, y_{2}\right)}}$ and

$$
I_{h_{0}}^{*}\left(T(P-\mathcal{K})_{e}(f)\right)=\left\langle\frac{\partial h_{0}}{\partial x}\right\rangle_{\mathcal{E}_{\left(x, y_{2}\right)}}+\left\langle 1, \frac{\partial h_{0}}{\partial y_{2}}\right\rangle_{I_{h_{0}}^{*} \mathcal{E}_{\left(y_{1}, y_{2}\right)}}
$$


We now verify the following equality

$$
\begin{aligned}
\mathcal{E}_{\left(x, y_{2}\right)} \times\{0\} \cap T(\mathcal{A})_{e}\left(g_{f}\right)= & \left\langle\left(\frac{\partial h_{0}}{\partial x}, 0\right)\right\rangle_{\mathcal{E}_{\left(x, y_{2}\right)}} \\
& +\left\langle(1,0),\left(\frac{\partial h_{0}}{\partial y_{2}}, 0\right)\right\rangle_{I_{h_{0}^{*}} \mathcal{E}_{\left(y_{1}, y_{2}\right)}}
\end{aligned}
$$

By the definition of $T(\mathcal{A})_{e}\left(g_{f}\right)$ and the equality (4), we may assume that any $(\zeta, 0) \in \mathcal{E}_{\left(x, y_{2}\right)} \times\{0\} \cap T(\mathcal{A})_{e}\left(g_{f}\right)$ has the form

$$
(\zeta, 0)=\left(\xi \frac{\partial h_{0}}{\partial x}, 0\right)+\left(\lambda \frac{\partial h_{0}}{\partial y_{2}}, \lambda\right)+\left(I_{h_{0}}^{*} \eta_{1}, I_{h_{0}}^{*} \eta_{2}\right)
$$

for some $\eta_{1}, \eta_{2} \in \mathcal{E}_{\left(y_{1}, y_{2}\right)}$ and $\xi, \lambda \in \mathcal{E}_{\left(x, y_{2}\right)}$. Hence $(\zeta, 0)=\left(\xi \frac{\partial h_{0}}{\partial x}-\left(I_{h_{0}}^{*} \eta_{2}\right)\right.$ $\left.\frac{\partial h_{0}}{\partial y_{2}}+\left(I_{h_{0}}^{*} \eta_{1}\right) \cdot 1,0\right) \in\left\langle\left(\frac{\partial h_{0}}{\partial x}, 0\right)\right\rangle_{\mathcal{E}_{\left(x, y_{2}\right)}}+\left\langle\left(\frac{\partial h_{0}}{\partial y_{2}}, 0\right),(1,0)\right\rangle_{I_{h_{0}}^{*} \mathcal{E}_{\left(y_{1}, y_{2}\right)}}$, that is $(\zeta, 0) \in$ the right hand side of $(5)$. The converse can be verified similarly, so we omit its proof.

By (4) and (5), we have

$$
\begin{aligned}
\mathcal{E}_{\left(x, y_{2}\right)} \times\{0\} & \cap T(\mathcal{A})_{e}\left(g_{f}\right) \\
= & \left\langle\left(\frac{\partial h_{0}}{\partial x}, 0\right)\right\rangle_{\mathcal{E}_{\left(x, y_{2}\right)}}+\left\langle(1,0),\left(\frac{\partial h_{0}}{\partial y_{2}}, 0\right)\right\rangle_{I_{h_{0}}^{*} \mathcal{E}_{\left(y_{1}, y_{2}\right)}} \\
= & I_{h_{0}}^{*}\left(T(P-\mathcal{K})_{e}(f) \times\{0\}\right.
\end{aligned}
$$

Then

$$
\begin{aligned}
I_{h_{0}}^{*} T(P-\mathcal{K})_{e}(f) & \cong I_{h_{0}}^{*} T(P-\mathcal{K})_{e}(f) \times\{0\} \\
& =\mathcal{E}_{\left(x, y_{2}\right)} \times\{0\} \cap T(\mathcal{A})_{e}\left(g_{f}\right),
\end{aligned}
$$

and $I_{h_{0}}^{*}$ induces an $\mathbb{R}$-isomorphism :

$$
\begin{aligned}
\mathcal{E}_{\left(x, y_{1}, y_{2}\right)} / T(P-\mathcal{K})_{e}(f) & \cong \mathcal{E}_{\left(x, y_{2}\right)} \times\{0\} / \mathcal{E}_{\left(x, y_{2}\right)} \\
& \times\{0\} \cap T(\mathcal{A})_{e}\left(g_{f}\right) .
\end{aligned}
$$

On the other hand, since $g_{f}\left(x, y_{2}\right)=\left(h_{0}\left(x, y_{2}\right), y_{2}\right)$, it is clear that

$$
\mathcal{E}_{\left(x, y_{2}\right)} \times \mathcal{E}_{\left(x, y_{2}\right)}=\mathcal{E}_{\left(x, y_{2}\right)} \times\{0\}+T(\mathcal{A})_{e}\left(g_{f}\right) .
$$

Then

$$
\mathcal{E}_{\left(x, y_{1}, y_{2}\right)} / T(P-\mathcal{K})_{e}(f) \cong \mathcal{E}_{\left(x, y_{2}\right)} \times\{0\} / T(\mathcal{A})_{e}\left(g_{f}\right) \cap \mathcal{E}_{\left(x, y_{2}\right)} \times\{0\}
$$




$$
\begin{aligned}
& \cong \mathcal{E}_{\left(x, y_{2}\right)} \times\{0\}+T(\mathcal{A})_{e}\left(g_{f}\right) / T(\mathcal{A})_{e}\left(g_{f}\right) \\
& =\mathcal{E}_{\left(x, y_{2}\right)} \times \mathcal{E}_{\left(x, y_{2}\right)} / T(\mathcal{A})_{e}\left(g_{f}\right) .
\end{aligned}
$$

If $\mathcal{E}_{\left(x, y_{1}, y_{2}\right)}=T(P-\mathcal{K})_{e}(f)$, by the above equality we have $\mathcal{E}_{\left(x, y_{2}\right)} \times$ $\mathcal{E}_{\left(x, y_{2}\right)}=T(\mathcal{A})_{e}\left(g_{f}\right)$. Hence (i) holds if and only if (ii) holds. On the other hand, since

$$
\left.\frac{\partial G_{F}}{\partial t}\right|_{t=0}=\left(\left.\frac{\partial h}{\partial t}\right|_{t=0}, 0\right) \in \mathcal{E}_{\left(x, y_{2}\right)} \times \mathcal{E}_{\left(x, y_{2}\right)}
$$

and

$$
\left.\frac{\partial F}{\partial t}\right|_{t=0}=-\left.\frac{\partial h}{\partial t}\right|_{t=0} \in \mathcal{E}_{\left(x, y_{2}\right)},
$$

the condition

$$
\operatorname{dim}_{\mathbb{R}} \mathcal{E}_{\left(x, y_{1}, y_{2}\right)} / T(P-\mathcal{K})_{e}(f)=1
$$

is equivalent to

$$
\operatorname{dim}_{\mathbb{R}} \mathcal{E}_{\left(x, y_{2}\right)} \times \mathcal{E}_{\left(x, y_{2}\right)} / T(\mathcal{A})_{e}\left(g_{f}\right)=1 .
$$

In this case, $F$ is a $(P-\mathcal{K})$-versal deformation of $f$ if and only if $\left.\frac{\partial F}{\partial t}\right|_{t=0} \notin$ $T(P-\mathcal{K})_{e}(f)$. Moreover

$$
\begin{aligned}
I_{h_{0}}^{*}\left(\left.\frac{\partial F}{\partial t}\right|_{t=0}\right) & =I_{h_{0}}^{*}\left(\left.\frac{-\partial h}{\partial t}\right|_{t=0}\right) \\
& =\left(\left.\frac{-\partial h}{\partial t}\right|_{t=0}, 0\right)=\left.\frac{-\partial G_{F}}{\partial t}\right|_{t=0}
\end{aligned}
$$

so that

$$
\left.\frac{\partial F}{\partial t}\right|_{t=0} \notin T(P-\mathcal{K})_{e}(f) \text { if and only if }\left.\frac{-\partial G_{F}}{\partial t}\right|_{t=0} \notin T(\mathcal{A})_{e}\left(g_{f}\right) .
$$

The last condition is equivalent to $G_{F}$ is an $\mathcal{A}$-versal deformation of $g_{f}$. For the other case $\left(\frac{\partial F}{\partial y_{2}} \neq 0\right.$ or $\left.\frac{\partial F}{\partial x} \neq 0\right)$, the proof is similar.

\section{Generic property of shadows of the moving surface}

In this section we use Thom's $k$-transversal theorem to shows generic property of shadows of the moving surface.

Theorem 3.1 There exists a dense subset $\mathcal{Q} \subset \mathcal{P}$ such that for any e $\in \mathcal{Q}$ and $\left(p_{0}, t_{0}\right) \in H \times I$, the set germ of the shadow of $e(H \times I)$ at $\Pi \circ e\left(p_{0}, t_{0}\right)$ 
is $t$-diffeomorphic to one of the following normal forms ${ }^{p} G_{k}^{( \pm)}$:

$$
\begin{aligned}
{ }^{p} G_{k}^{( \pm)}= & \left\{\left(y_{1}, y_{2}, t\right) \in\left(\mathbb{R}^{2} \times \mathbb{R}, 0\right) \mid x^{k+1}+\sum_{i=1}^{k-1} y_{i} x^{i-1}\right. \\
& +p x^{k-1}\left(t \pm y_{k}^{2}\right)+(1-p) y_{k} x^{k-1}=0 \\
& \text { for some } x \in(\mathbb{R}, 0)\}
\end{aligned}
$$

where $p=0,1$, and $2 p \leqslant k \leqslant p+2$.

Proof. Take $\ell$ to be sufficiently large. Let $\hat{S}_{j}, j=0,1,2$ or 3 , be the set of jets $z=J^{1}(h)(0,0)$ of $J^{\ell}(2,2)$ with $(\mathcal{A})-\operatorname{cod}(h)=j$. Let $\Sigma$ be the compliment of $\cup_{j=0}^{3} \hat{S}_{j}$ in $J^{\ell}(2,2)$ (That is, $\Sigma$ is the union of jets $j^{\ell}(h)$ with $(\mathcal{A})-\operatorname{cod}(h) \geqslant 4)$. Then we have

$$
J^{\ell}(2,2)=\hat{S}_{0} \cup \hat{S}_{1} \cup \hat{S}_{2} \cup \hat{S}_{3} \cup \Sigma .
$$

Now we consider the subsets $S_{j}=H^{2} \times \mathbb{R}^{2} \times \hat{S}_{j}$ in $J^{\ell}\left(H, \mathbb{R}^{2}\right)$. For any $e \in \mathcal{P}$, we define the $\ell-j e t-$ extension map $j_{1}^{\ell} e: H \times I \rightarrow J^{\ell}\left(H, \mathbb{R}^{3}\right)$ given by

$$
j_{1}^{\ell} e(p, t):=j^{\ell}\left(i_{t}(p)\right)
$$

where $i_{t}=\left.e\right|_{H \times\{t\}}$.

We also consider the projection ${ }^{\ell} \pi: J^{\ell}\left(H, \mathbb{R}^{3}\right) \rightarrow J^{\ell}\left(H, \mathbb{R}^{2}\right)$ defined by

$$
{ }^{\ell} \pi\left(j^{\ell} h(x)\right)=j^{\ell}(\Pi \circ h(x))
$$

for $h:\left(H, p_{0}\right) \rightarrow\left(\mathbb{R}^{3}, h\left(p_{0}\right)\right)$ and $\Pi: \mathbb{R} \times \mathbb{R}^{2} \rightarrow \mathbb{R}^{2}$.

Since ${ }^{\ell} \pi$ is a submersion and $S_{j}(j=0,1,2,3)$ are submanifolds of $J^{\ell}\left(H, \mathbb{R}^{2}\right),{ }^{\ell} \pi^{-1}\left(S_{j}\right)$ are submanifolds in $J^{\ell}\left(H, \mathbb{R}^{3}\right)$ and

$$
\text { codim of } S_{j}=\text { codim of }{ }^{\ell} \pi^{-1}\left(S_{j}\right) \quad(j=0,1,2,3) .
$$

Moreover, we can show that

$$
j_{1}^{\ell}(e) 历{ }^{\ell} \pi^{-1}\left(S_{j}\right) \text { if and only if } j_{1}^{\ell}(\Pi \circ e) 历 S_{j} .
$$

Set

$$
\hat{\mathcal{Q}}_{j}:=\left\{e \in \mathcal{P} \mid j_{1}^{\ell}(e) \bar{\pitchfork}^{\ell} \pi^{-1}\left(S_{j}\right)\right\}, \quad(j=0,1,2,3) .
$$


and

$$
\mathcal{Q}_{\Sigma}:=\left\{e \in \mathcal{P} \mid j_{1}^{\ell}(e) \cap^{\ell} \pi^{-1}(\Sigma)=\phi\right\},
$$

By [11], $\mathcal{Q}_{\Sigma}$ is an algebraic subset of $J^{\ell}(2,2)$ of codimension $\geqslant 4$.

It follows from Thom's $k$-transversal Theorem (See [10]) that $\hat{\mathcal{Q}}_{j}$ are residual subsets of $\mathcal{P}$.

Finally we set

$$
\mathcal{Q}=\left(\cap_{j=0}^{3} \hat{\mathcal{Q}}_{j}\right) \cap \mathcal{Q}_{\Sigma} \subset \mathcal{P}
$$

then $\mathcal{Q}$ is a residual subset in $\mathcal{P}$.

For any $e \in \mathcal{Q}$ and $\left(p_{0}, t_{0}\right) \in H \times I$, there exists a neighbourhood $U$ of $e\left(p_{0}, t_{0}\right)$ and a local equation $F:\left(U, e\left(p_{0}, t_{0}\right)\right) \rightarrow(\mathbb{R}, 0)$ of $e$ at $e\left(p_{0}, t_{0}\right)$, so that $F^{-1}(0)=U \cap e(H \times I)$. Without the loss of generality, $e\left(p_{0}, t_{0}\right)$ is assumed to be the origin, so that we consider a submersion germ $F$ : $\left(\mathbb{R} \times \mathbb{R}^{2} \times \mathbb{R}, 0\right) \rightarrow(\mathbb{R}, 0)$. Under the above notation, we may have the following identification :

$$
j_{1}^{\ell} \Pi \circ e=j_{1}^{\ell} \pi_{2} \circ \Pi_{F} .
$$

Since $e \in \mathcal{Q}, j_{1}^{\ell} \pi_{2} \circ \Pi_{F}$ is transversal to $S_{j}$. It follows from lemma 2.6, that $\pi_{2} \circ \Pi_{F}$ is an $\mathcal{A}$-versal deformation of $f$. Moreover, by the Proposition $2.7 F$ is $P-\mathcal{K}$-versal deformation of $f=\left.F\right|_{\mathbb{R} \times \mathbb{R}^{2} \times\left\{t_{0}\right\}}$. Hence we may apply Corollary 2.5 to get the result.

\section{Classification of multi-shadows of the moving surface}

In this section, we consider the local shadows of a generic submanifold $e(H \times I)$ in $\mathbb{R}^{3} \times \mathbb{R}$ around $r$-points $e\left(p_{1}, t_{0}\right), \ldots, e\left(p_{r}, t_{0}\right)$. The all results in this section are the multi-germ version of results in $\S 2,3$, so that we omit the detail of the proofs.

Let $e \in \mathcal{P}$ and $p_{1}, \ldots, p_{r} \in H$, where $p_{i} \neq p_{j}$ as $i \neq j$, for $i, j=1, \ldots, r$, and let $e_{i}:\left(H \times I,\left(p_{i}, t_{0}\right)\right) \rightarrow\left(\mathbb{R} \times \mathbb{R}^{2} \times \mathbb{R},\left(x_{i}, 0,0\right)\right)$ be the germ of $e$ at $\left(p_{i}, t_{0}\right)(i=1, \ldots, r)$. The $r$-multiple $e_{1} \times \ldots \times e_{r}$ is called an $r$-multi-germ of $e \in \mathcal{P}$ at $\left(p_{1}, t_{0}\right), \ldots,\left(p_{r}, t_{0}\right)$.

As in $\S 2$, for each $e_{i}$, there exists a neighborhood $U_{i}$ of $e\left(p_{i}, t_{0}\right)$ in $\mathbb{R} \times \mathbb{R}^{2} \times \mathbb{R}$ and a local equation $F_{i}:\left(U_{i}, e\left(p_{i}, t_{0}\right)\right) \rightarrow(\mathbb{R}, 0)$ such that $F_{i}^{-1}(0)=U_{i} \cap e(H \times I)$. The $r$-multiple $F_{1} \times \ldots \times F_{r}$ is called an $r$-multi-local equation of the $r$-multi-germ $e_{1} \times \ldots \times e_{r}$ of $e \in \mathcal{P}$ at $\left(p_{1}, t_{0}\right), \ldots,\left(p_{r}, t_{0}\right)$. The 
$r$-multi-local equation $F_{1} \times \ldots \times F_{r}$ express the properties of the submanifold $e(H \times I)$ around $r$ points $e\left(p_{1}, t_{0}\right), \ldots, e\left(p_{r}, t_{0}\right)$.

We first consider the local case.

Definition 4.1 Let $F_{i}$ and $F_{i}^{\prime}:\left(\mathbb{R} \times \mathbb{R}^{2} \times \mathbb{R}, 0\right) \rightarrow(\mathbb{R}, 0)$ be function germs $(i=1, \ldots, r)$. We say that $F_{1} \times \ldots \times F_{r}$ and $F_{1}^{\prime} \times \ldots \times F_{r}^{\prime}$ are $t-(P-\mathcal{K})_{r}-$ equivalent if there exists a diffeomorphism germ

$$
\Phi_{i}:\left(\mathbb{R} \times \mathbb{R}^{2} \times \mathbb{R}, 0\right) \rightarrow\left(\mathbb{R} \times \mathbb{R}^{2} \times \mathbb{R}, 0\right) \quad i=1, \ldots, r
$$

of the form

$$
\Phi_{i}\left(x, y_{1}, y_{2}, t\right)=\left(\phi_{1}^{i}\left(x, y_{1}, y_{2}, t\right), \phi_{2}\left(y_{1}, y_{2}, t\right), \phi_{3}(t)\right)
$$

such that

$$
\Phi_{i}^{*}\left\langle F_{i}^{\prime}\right\rangle_{\mathcal{E}\left(x, y_{1}, y_{2}, t\right)}=\left\langle F_{i}\right\rangle_{\mathcal{E}\left(x, y_{1}, y_{2}, t\right)}, \quad i=1, \ldots, r
$$

Similarly we can define $(P-\mathcal{K})_{r}$-equivalence for $f_{1} \times \ldots \times f_{r}=F_{1} \times$ $\ldots \times\left. F_{r}\right|_{t=0}($ See $[6],[7])$.

Firstly, we give tools for classifications of multi-germs under the $t-$ $(P-\mathcal{K})_{r}$-equivalence. We consider the subset of $\mathcal{E}_{\left(x, y_{1}, y_{2}\right)}^{r}=\mathcal{E}_{\left(x, y_{1}, y_{2}\right)} \times \ldots \times$ $\mathcal{E}_{\left(x, y_{1}, y_{2}\right)}$ given by

$$
\begin{aligned}
T(P-\mathcal{K})_{r}\left(f_{1} \times \ldots \times f_{r}\right) \\
=\left\langle\frac{\partial f_{1}}{\partial x}\right\rangle_{\mathfrak{M}_{\left(x, y_{1}, y_{2}\right)} \times \ldots \times\left\langle\frac{\partial f_{r}}{\partial x}\right\rangle_{\mathfrak{M}_{\left(x, y_{1}, y_{2}\right)}}} \times \ldots \times\left\langle f_{r}\right\rangle_{\mathcal{E}_{\left(x, y_{1}, y_{2}\right)}} \\
\left.\quad+\left\langle f_{1}\right\rangle_{\left.\mathcal{E}_{\left(x, y_{1}, y_{2}\right)} \times \ldots f_{r}\right)}\right\rangle_{\mathfrak{M}_{\left(y_{1}, y_{2}\right)}} \\
\quad+\left\langle\frac{\partial\left(f_{1} \times \ldots \times f_{r}\right)}{\partial y_{1}}, \frac{\partial\left(f_{1} \times \ldots \times y_{2}\right.}{}\right.
\end{aligned}
$$

and define

$$
\begin{aligned}
& (P-\mathcal{K})_{r}-\operatorname{cod}\left(f_{1} \times \ldots \times f_{r}\right) \\
& \quad=\operatorname{dim}_{\mathbb{R}} \mathfrak{M}_{\left(x, y_{1}, y_{2}\right)}^{r} / T(P-\mathcal{K})_{r}\left(f_{1} \times \ldots \times f_{r}\right)
\end{aligned}
$$

where

$$
\mathfrak{M}_{\left(x, y_{1}, y_{2}\right)}^{r}=\mathfrak{M}_{\left(x, y_{1}, y_{2}\right)} \times \ldots \times \mathfrak{M}_{\left(x, y_{1}, y_{2}\right)}
$$

We also consider the subset of $\mathcal{E}_{\left(x, y_{1}, y_{2}\right)}^{r}=\mathcal{E}_{\left(x, y_{1}, y_{2}\right)} \times \ldots \times \mathcal{E}_{\left(x, y_{1}, y_{2}\right)}$ given 
by

$$
\begin{aligned}
& T(\left.P-\mathcal{K})_{e}\right)_{r}\left(f_{1} \times \ldots \times f_{r}\right) \\
&=\langle\left.\left.\frac{\partial f_{1}}{\partial x}, f_{1}\right\rangle_{\left.\mathcal{E}_{\left(x, y_{1}, y_{2}\right)} \times \ldots \times f_{r}\right)}, \frac{\partial\left(f_{1} \times \ldots \times f_{r}\right)}{\partial x}, f_{r}\right\rangle_{\mathcal{E}_{\left(x, y_{1}, y_{2}\right)}} \\
& \quad+\left\langle\frac{\partial\left(f_{1} \times \ldots y_{2}\right.}{\partial y_{1}}\right\rangle_{\mathcal{E}_{\left(y_{1}, y_{2}\right)}}
\end{aligned}
$$

and define

$$
\begin{aligned}
& \left((P-\mathcal{K})_{e}\right)_{r}-\operatorname{cod}\left(f_{1} \times \ldots \times f_{r}\right) \\
& \quad=\operatorname{dim}_{\mathbb{R}} \mathcal{E}_{\left(x, y_{1}, y_{2}\right)}^{r} / T\left((P-\mathcal{K})_{e}\right)_{r}\left(f_{1} \times \ldots \times f_{r}\right) .
\end{aligned}
$$

We call that $F_{1} \times \ldots \times F_{r}$ is the $(P-\mathcal{K})_{r}$-versal deformation of $f_{1} \times \ldots \times f_{r}$ if

$$
\begin{aligned}
\mathcal{E}_{\left(x, y_{1}, y_{2}\right)}^{r}= & \left\langle\left.\frac{\partial\left(F_{1} \times \ldots \times F_{r}\right)}{\partial t}\right|_{\mathbb{R} \times \mathbb{R}^{2} \times\{0\}}\right\rangle_{\mathbb{R}} \\
& +T\left((P-\mathcal{K})_{e}\right)_{r}\left(f_{1} \times \ldots \times f_{r}\right) .
\end{aligned}
$$

Similarly we may define $T(\mathcal{K})_{r}\left(f_{0,1} \times \ldots \times f_{0, r}\right),(\mathcal{K})_{r}-\operatorname{cod}\left(f_{0,1} \times \ldots \times\right.$ $\left.f_{0, r}\right)$ and $(\mathcal{K})_{r}$-versal deformation of $f_{0,1} \times \ldots \times f_{0, r}$, where $f_{0, i}=\left.f_{i}\right|_{\mathbb{R} \times\{0\} \times\{0\}}$ and $i=1, \ldots, r$ (See [2],[3], [9]).

Lemma 4.2 $\sum_{i=1}^{r} \mathcal{K}-\operatorname{cod}\left(f_{0, i}\right) \leqslant(P-\mathcal{K})_{r}-\operatorname{cod}\left(f_{1} \times \ldots \times f_{r}\right) \leqslant((P-$ $\left.\mathcal{K})_{e}\right)_{r}-\operatorname{cod}\left(f_{1} \times \ldots \times f_{r}\right)+2$.

Where

$$
\mathcal{K}-\operatorname{cod}\left(f_{0, i}\right)=\operatorname{dim}_{\mathbb{R}} \mathfrak{M}_{x} /\left\langle\frac{\partial f_{0, i}}{\partial x}\right\rangle_{\mathfrak{M}_{x}}+\left\langle f_{0, i}\right\rangle_{\mathcal{E}_{x}}
$$

Like as in the case when $r=1$, Zakalyukin's theorem $([13])$ is again the key of our classification.

In order to state Zakalyukin's theorem we define the discriminant set of $r$-multi germs $G_{1} \times \ldots \times G_{r}$, where $G_{i}:\left(\mathbb{R} \times \mathbb{R}^{2} \times \mathbb{R}, 0\right) \rightarrow(\mathbb{R}, 0)$ are function-germs $(i=1, \ldots, r)$ as follows :

$$
D_{G}=\cup_{i=1}^{r} D_{G_{i}},
$$

where

$$
D_{G_{i}}=\left\{u \in \mathbb{R}^{2} \times \mathbb{R} \mid G_{i}\left(x, u_{1}, u_{2}, u_{3}\right)\right.
$$




$$
\begin{gathered}
=\frac{\partial G_{i}}{\partial u_{1}}(x, u)=\frac{\partial G_{i}}{\partial u_{2}}(x, u)=\frac{\partial G_{i}}{\partial u_{3}}(x, u)=0, \\
\text { for some } x \in(\mathbb{R}, 0)\} .
\end{gathered}
$$

We will utilize the following result for $n=2$.

Proposition 4.3 (Zakalyukin's Theorem $[13])$ Let $t:\left(\mathbb{R}^{3}, 0\right) \rightarrow(\mathbb{R}, 0)$ be a submersion germ which is defined on the $\left(u_{11}, \ldots, u_{1 m_{1}}, \ldots, u_{r 1}, \ldots, u_{r m_{r}}\right.$, $\left.u_{1}, \ldots, u_{\mu}\right)$-space, where $\mu=3-\sum_{i=1}^{r} m_{i}$. Suppose that

$$
\frac{\partial t}{\partial u_{1 m_{1}}} \neq 0, \ldots, \frac{\partial t}{\partial u_{r m_{r}}} \neq 0
$$

and

$$
\left.t\right|_{u_{11}=\ldots=u_{r m_{r}}=0}
$$

is a Morse function germ. Then there exists a diffeomorphism germ $\phi$ : $\left(\mathbb{R}^{3}, 0\right) \rightarrow\left(\mathbb{R}^{3}, 0\right)$ preserving the discriminant set $D_{F}$ such that $t \circ \phi=u_{1}$ or $t \circ \phi= \pm u_{1 m_{1}} \pm \ldots \pm u_{r m_{r}} \pm u_{1}^{2} \pm \ldots \pm u_{\mu}^{2}$.

Remark. The submersion $t$ which satisfies the assumption of the Proposition 4.3 is generic.

If $\mathcal{K}-\operatorname{cod}\left(f_{0, i}\right)=0$, then the germ $f_{0, i}$ is non-singular, so that the image of the neighbourhood of such a point by the projection $\Pi$ is contained in the inside of shadows. Hence, we need only consider germs with $\mathcal{K}-\operatorname{cod}\left(f_{0, i}\right) \geqslant$ 1. Moreover, since we will classify multi-germs with $\left((P-\mathcal{K})_{e}\right)_{r}-\operatorname{cod}\left(f_{1} \times\right.$ $\left.\ldots \times f_{r}\right) \leqslant 1$ (See [7]), we may assume that $r \leqslant 3$ by Lemma 4.2 .

We can prove the following multi-germ version of Theorem 2.3 by exactly in the same way as the single germ case.

Theorem 4.4 Let $F_{i}:\left(\mathbb{R} \times \mathbb{R}^{2} \times \mathbb{R}, 0\right) \rightarrow(\mathbb{R}, 0)$ be function germs with $\left((P-\mathcal{K})_{e}\right)_{r}-\operatorname{cod}\left(f_{1} \times \ldots \times f_{r}\right) \leqslant 1$, where $f_{i}=\left.F_{i}\right|_{\mathbb{R} \times \mathbb{R}^{2} \times\{0\}}$ for $i=1, \ldots, r$ and $1<r \leqslant 3$. If $F_{1} \times \ldots \times F_{r}$ is a $\left((P-\mathcal{K})_{e}\right)_{r}$-versal deformation of $f_{1} \times \ldots \times f_{r}$, then $F_{1} \times \ldots \times F_{r}$ is $t-(P-\mathcal{K})_{r}$-equivalent to one of the following $r$-multi-germs :

$$
\begin{aligned}
& { }_{2}^{0} A_{1,1}:\left(x^{2}+y_{1}, x^{2}+y_{2}\right) \\
& { }_{2}^{1} A_{1,1}^{+}:\left(x^{2}+t+y_{1}+y_{2}^{2}, x^{2}+y_{1}\right) \\
& { }_{2}^{1} A_{1,1}^{-}:\left(x^{2}+t-y_{1}-y_{2}^{2}, x^{2}+y_{1}\right)
\end{aligned}
$$




$$
\begin{aligned}
& { }_{2}^{1} A_{2,1}^{+}:\left(x^{3}+t x+x y_{2}+y_{1}, x^{2}+y_{2}\right) \\
& { }_{2}^{1} A_{2,1}^{-}:\left(x^{3}+t x-x y_{2}+y_{1}, x^{2}+y_{2}\right) \\
& { }_{2}^{1} A_{1,2}^{+}:\left(x^{2}+t+y_{2}, x^{3}+x y_{2}+y_{1}\right) \\
& { }_{2}^{1} A_{1,2}^{-}:\left(x^{2}+t-y_{2}, x^{3}+x y_{2}+y_{1}\right) \\
& { }_{3}^{1} A_{1,1,1}^{+}:\left(x^{2}+t+y_{1}+y_{2}, x^{2}+y_{1}, x^{2}+y_{2}\right) \\
& { }_{3}^{1} A_{1,1,1}^{-}:\left(x^{2}+t-y_{1}-y_{2}, x^{2}+y_{1}, x^{2}+y_{2}\right) .
\end{aligned}
$$

We can denote the above list to the following form

$$
{ }_{r}^{p} A_{k_{1} k_{2} k_{3}}^{( \pm)}=\left({ }^{p} A_{k_{1}},{ }^{p} A_{k_{2}},{ }^{p} A_{k_{3}}\right)
$$

where

$$
\begin{aligned}
{ }^{p} A_{k_{1}}= & x^{k_{1}+1}+\sum_{i=1}^{k_{1}-1} y_{i} x^{i-1} \\
& +p x^{k_{1}-1}\left(t \pm y_{k_{1}+k_{2}-1} \pm y_{k_{1}+k_{2}+k_{3}-1} \pm y_{k_{1}+k_{2}+k_{3}}^{2}\right) \\
& +(1-p) y_{k_{1}} x^{k_{1}-1}, \\
{ }^{p} A_{k_{2}}= & x^{k_{2}+1}+p \sum_{i=1}^{k_{1}+k_{2}-1} y_{k_{1}-1+i} x^{i-1} \\
& +(1-p) \sum_{i=1}^{k_{1}+k_{2}-1} y_{k_{1}+i} x^{i-1}, \\
p_{k_{3}=} & x^{k_{3}+1}+p \sum_{i=1}^{k_{1}+k_{2}+k_{3}-1} y_{k_{1}+k_{2}-1+i} x^{i-1} \\
& +(1-p) \sum_{i=1}^{k_{1}+k_{2}+k_{3}-1} y_{k_{1}+k_{2}+i} x^{i-1},
\end{aligned}
$$

for $p=0,1 . \quad r=2,3 . \quad r \leqslant \sum_{i=1}^{r} k_{i} \leqslant 3$ and $y_{k_{1}+k_{2}-1}=0$ if $k_{1} k_{2}=0$, $y_{k_{1}+k_{2}+k_{3}-1}=0$ if $k_{1} k_{2} k_{3}=0,{ }^{p} A_{k_{i+1}}=0$ if $i=r$.

Proposition 4.5 If $F_{1} \times \ldots \times F_{r}, F_{1}^{\prime} \times \ldots \times F_{r}^{\prime}$ are $t-(P-\mathcal{K})$-equivalent, then $\Pi\left(\cup_{i=1}^{r} F_{i}^{-1}(0)\right), \Pi\left(\cup_{i=1}^{r} F_{i}^{\prime-1}(0)\right)$ are $t$-diffeomorphic, where $\Pi: \mathbb{R} \times$ $\mathbb{R}^{2} \times \mathbb{R} \rightarrow \mathbb{R}^{2} \times \mathbb{R}$ is the canonical projection.

As a corollary of Theorem 4.4 and Proposition 4.5 we have the following result. 
Corollary 4.6 Under the same assumptions of Theorem 4.4, $\Pi\left(\cup_{i=1}^{r}\right.$ $\left.F_{i}^{-1}(0)\right)$ is t-diffeomorphism to one of the germs in the following list:

$$
{ }_{2}^{0} G_{1,1} \quad{ }_{2}^{1} G_{1,1}^{+} \quad{ }_{2}^{1} G_{1,1}^{-} \quad{ }_{2}^{1} G_{2,1}^{+} \quad{ }_{2}^{1} G_{2,1}^{-} \quad{ }_{2}^{1} G_{1,2}^{+} \quad{ }_{2}^{1} G_{1,2}^{-} \quad{ }_{3}^{1} G_{1,1,1}^{+} \quad{ }_{3}^{1} G_{1,1,1}^{-},
$$

where

$$
{ }_{2}^{p} G_{k_{1}, k_{2}}^{( \pm)}=\Pi\left({ }^{p} A_{k_{1}}=0\right) \cup \Pi\left({ }^{p} A_{k_{2}}=0\right),
$$

and

$$
{ }_{3}^{p} G_{k_{1}, k_{2}, k_{3}}^{( \pm)}=\Pi\left({ }^{p} A_{k_{1}}=0\right) \cup \Pi\left({ }^{p} A_{k_{2}}=0\right) \cup \Pi\left({ }^{p} A_{k_{3}}=0\right)
$$

for $p=0,1$, and $2 \leqslant \sum_{i=1}^{r} k_{i} \leqslant 3$.

In order to show that the list in corollary 4.5 is a generic local classification of shadows of $e$, we need some preparations.

Let $g_{i}, g_{i}^{\prime}:\left(\mathbb{R}^{2}, 0\right) \rightarrow\left(\mathbb{R}^{2}, 0\right)$ be map-germs $(i=1, \ldots, r)$. We say that $g_{1} \times \ldots \times g_{r}$ and $g_{1}^{\prime} \times \ldots \times g_{r}^{\prime}$ are $\mathcal{A}_{r, 1}$-equivalent if there exist diffeomorphism germs

$$
\Phi, \Psi_{i}:\left(\mathbb{R}^{2}, 0\right) \rightarrow\left(\mathbb{R}^{2}, 0\right) \quad(i=1, \ldots, r)
$$

such that

$$
\Phi \circ g_{i}=g_{i}^{\prime} \circ \Psi_{i} . \quad \text { for } i=1, \ldots, r .
$$

We define the tangent space of $\mathcal{A}_{r, 1}$-orbit of $g_{1} \times \ldots \times g_{r}$ is follows.

$$
\begin{aligned}
T(\mathcal{A})_{r, 1}\left(g_{1} \times \ldots \times g_{r}\right)= & \left\langle\frac{\partial g_{1}}{\partial x_{1}}, \frac{\partial g_{1}}{\partial x_{2}}\right\rangle_{\mathfrak{M}_{2}} \times \ldots \times\left\langle\frac{\partial g_{r}}{\partial x_{1}}, \frac{\partial g_{r}}{\partial x_{2}}\right\rangle_{\mathfrak{M}_{2}} \\
& +g^{(r *)} \triangle_{r}\left(\mathfrak{M}_{2} \times \mathfrak{M}_{2}\right)
\end{aligned}
$$

where

$$
g^{(r *)}=\left(g_{1}^{*} \times g_{1}^{*}\right) \times \ldots \times\left(g_{r}^{*} \times g_{r}^{*}\right):\left(\mathcal{E}_{2} \times \mathcal{E}_{2}\right)^{r} \rightarrow\left(\mathcal{E}_{2} \times \mathcal{E}_{2}\right)^{r},
$$

and

$$
\begin{aligned}
& \triangle_{r}\left(\mathfrak{M}_{2} \times \mathfrak{M}_{2}\right) \\
& \quad=\left\{\left(\left(h_{1}, h_{2}\right), \ldots,\left(h_{1}, h_{2}\right)\right) \in\left(\mathfrak{M}_{2} \times \mathfrak{M}_{2}\right)^{r} \mid h_{1}, h_{2} \in \mathfrak{M}_{2}\right\}
\end{aligned}
$$

We also consider another notion of codimensions of $\left(g_{1} \times \ldots \times g_{r}\right)$ defined as follows :

$$
\mathcal{A}_{r, 1}-\operatorname{cod}\left(g_{1} \times \ldots \times g_{r}\right)
$$




$$
=\operatorname{dim}_{\mathbb{R}}\left(\mathfrak{M}_{2} \times \mathfrak{M}_{2}\right)^{r} / T(\mathcal{A})_{r}\left(g_{1} \times \ldots \times g_{r}\right) .
$$

Similarly, we define the tangent space of $\left(\mathcal{A}_{e}\right)_{r, 1}$-orbit of $\left(g_{1} \times \ldots \times g_{r}\right)$ is follows :

$$
\begin{aligned}
T\left(\mathcal{A}_{e}\right)_{r, 1}\left(g_{1} \times \ldots \times g_{r}\right)= & \left\langle\frac{\partial g_{1}}{\partial x_{1}}, \frac{\partial g_{1}}{\partial x_{2}}\right\rangle_{\mathcal{E}_{2}} \times \ldots \times\left\langle\frac{\partial g_{r}}{\partial x_{1}}, \frac{\partial g_{r}}{\partial x_{2}}\right\rangle_{\mathcal{E}_{2}} \\
& +g^{(r *)} \triangle_{r}\left(\mathcal{E}_{2} \times \mathcal{E}_{2}\right),
\end{aligned}
$$

where

$$
\triangle_{r}\left(\mathcal{E}_{2} \times \mathcal{E}_{2}\right)=\left\{\left(\left(h_{1}, h_{2}\right), \ldots,\left(h_{1}, h_{2}\right)\right) \in\left(\mathcal{E}_{2} \times \mathcal{E}_{2}\right)^{r} \mid h_{1}, h_{2} \in \mathcal{E}_{2}\right\} .
$$

The codimension is defined by

$$
\begin{aligned}
& \left(\mathcal{A}_{e}\right)_{r, 1}-\operatorname{cod}\left(g_{1} \times \ldots \times g_{r}\right) \\
& \quad=\operatorname{dim}_{\mathbb{R}}\left(\mathcal{E}_{2} \times \mathcal{E}_{2}\right)^{r} / T\left(\mathcal{A}_{e}\right)_{r, 1}\left(g_{1} \times \ldots \times g_{r}\right) .
\end{aligned}
$$

Remark. $\quad T(\mathcal{A})_{r, 1}\left(g_{1} \times \ldots \times g_{r}\right)$ and $T\left((\mathcal{A})_{e}\right)_{r, 1}\left(g_{1} \times \ldots \times g_{r}\right)$ depend only on the equivalent class under the $\mathcal{A}_{r, 1}$-equivalence.

We say that $G_{1} \times \ldots \times G_{r}$ is an $\mathcal{A}_{r, 1}$-versal deformation of $g_{1} \times \ldots \times g_{r}$ if

$$
\left\langle\left.\frac{\partial\left(G_{1} \times \ldots \times G_{r}\right)}{\partial t}\right|_{t=0}\right\rangle_{\mathbb{R}}+T\left(\mathcal{A}_{e}\right)_{r, 1}\left(g_{1} \times \ldots \times g_{r}\right)=\mathcal{E}_{2} \times \mathcal{E}_{2} .
$$

Let $F_{i}:\left(\mathbb{R} \times \mathbb{R}^{2} \times \mathbb{R}, 0\right) \rightarrow(\mathbb{R}, 0)$ be a submersion-germ, for each $i=1, \ldots, r$. Let $f_{1} \times \ldots \times f_{r}=F_{1} \times \ldots \times\left. F_{r}\right|_{\mathbb{R} \times \mathbb{R}^{2} \times\{0\}}$. We consider local projections

$$
\Pi_{F_{i}}=\left.\Pi\right|_{F_{i}^{-1}(0)}:\left(F_{i}^{-1}(0), 0\right) \rightarrow\left(\mathbb{R}^{2} \times \mathbb{R}, 0\right)
$$

defined by

$$
\Pi_{F_{i}}\left(x, y_{1}, y_{2}, t\right)=\left(y_{1}, y_{2}, t\right), \quad \text { for } i=1, \ldots, r,
$$

and

$$
\pi_{f_{i}}=\left.\pi\right|_{f_{i}^{-1}(0)}:\left(f_{i}^{-1}(0), 0\right) \rightarrow\left(\mathbb{R}^{2}, 0\right)
$$

defined by

$$
\pi_{f_{i}}\left(x, y_{1}, y_{2}\right)=\left(y_{1}, y_{2}\right), \quad \text { for } i=1, \ldots, r .
$$


By the above remark, $T(\mathcal{A})_{r, 1}\left(\pi_{f_{1}} \times \ldots \times \pi_{f_{r}}\right)$ and $T\left((\mathcal{A})_{e}\right)_{r, 1}\left(\pi_{f_{1}} \times \ldots \times\right.$ $\pi_{f_{r}}$ ) are well-defined.

Proposition 4.7 The following conditions are equivalent.

(i) $F_{1} \times \ldots \times F_{r}$ is $(P-\mathcal{K})_{r}$-versal deformation of $f_{1} \times \ldots \times f_{r}$.

(ii) $\Pi_{F_{1}} \times \ldots \times \Pi_{F_{r}}$ is $\mathcal{A}_{r, 1}$-versal deformation of $\pi_{f_{1}} \times \ldots \times \pi_{f_{r}}$.

Let $G_{i}:\left(\mathbb{R}^{2} \times \mathbb{R}, 0\right) \rightarrow\left(\mathbb{R}^{2}, 0\right)$ be map-germs for $i=1, \ldots, r$ and $g_{i}=\left.G_{i}\right|_{\mathbb{R}^{2} \times\{0\}}$. Now we consider a map germ

$$
\begin{aligned}
j_{1}^{\ell} G:\left(\mathbb{R}^{2} \times\right. & \left.\ldots \times \mathbb{R}^{2} \times \mathbb{R}, 0\right) \\
& \rightarrow J^{\ell}\left(\mathbb{R}^{2}, \mathbb{R}^{2}\right) \times \ldots \times J^{\ell}\left(\mathbb{R}^{2}, \mathbb{R}^{2}\right) \quad(r \text { - times })
\end{aligned}
$$

given by

$$
j_{1}^{\ell} G\left(x_{1}, \ldots, x_{r}, t\right):=\left(j^{\ell} G_{1, t}\left(x_{1}\right), \ldots, j^{\ell} G_{r, t}\left(x_{r}\right)\right)
$$

and the canonical projection

$$
\begin{aligned}
\pi: J^{\ell}\left(\mathbb{R}^{2}, \mathbb{R}^{2}\right) \times \ldots \times J^{\ell}\left(\mathbb{R}^{2}, \mathbb{R}^{2}\right) & \\
& \rightarrow J^{\ell}(2,2) \times \ldots \times J^{\ell}(2,2) \quad(r-\text { times }) .
\end{aligned}
$$

Set $z_{i}=j^{\ell} g_{i}(0)$ for $i=1, \ldots, r$.

Proposition 4.8 Suppose that $\left(\mathcal{A}_{e}\right)_{r, 1}-\operatorname{cod}\left(g_{1} \times \ldots \times g_{r}\right)<+\infty$. For sufficiently large $\ell$, the following conditions are equivalent.

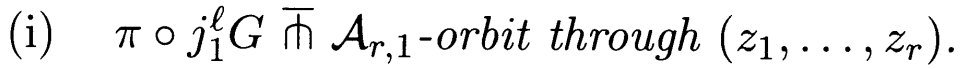

(ii) $G_{1} \times \ldots \times G_{r}$ is an $\mathcal{A}_{r, 1}$-versal deformation of $g_{1} \times \ldots \times g_{r}$, where $G\left(x_{1}, \ldots, x_{r}, t\right)=\left(G_{1}\left(x_{1}, t\right), \ldots, G_{r}\left(x_{r}, t\right)\right)$.

Theorem 4.9 There exists a residual subset $\mathcal{Q} \subset \mathcal{P}$ with the following property: For any $e \in \mathcal{Q}$ and for any point $Y_{0} \in \Pi \circ e(H \times I)$, the set germ of the shadow $\Pi \circ e(H \times I)$ at $Y_{0}$ is t-diffeomorphic to one of the germs in the list in Corollary 4.6.

Proof. We apply Thom's multi-jet transversality theorem (c.f., [3]) like as the proof of the Theorem 3.1. Let $\hat{S}_{j}$ be the $\mathcal{A}$-orbit through $z=j^{\ell} h(0)$ in $J^{\ell}(2,2)$ with $\mathcal{A}-\operatorname{cod}(h)=j$, where $j=0,1,2,3$. we consider a submanifold

$$
S\left(j_{1}, \ldots, j_{r}\right)=\left(\mathbb{R}^{2}\right)^{(r)} \times \triangle_{r}\left(\mathbb{R}^{2}\right) \times \hat{S}_{j_{1}} \times \ldots \times \hat{S}_{j_{r}}
$$

in the multi-jet space ${ }_{r} J^{\ell}\left(\mathbb{R}^{2}, \mathbb{R}^{2}\right)$, where $\triangle_{r}\left(\mathbb{R}^{2}\right)=\left\{(X, \ldots, X) \in\left(\mathbb{R}^{2}\right)^{r}\right.$ $\left.\mid X \in \mathbb{R}^{2}\right\}$. By the remark after Proposition 4.3, we may assume that 
$r=1,2,3$. We have already proved for the case $r=1$ by Theorem 3.1, so that we consider the case $r=2,3$. We remark that

$$
\operatorname{codim} S\left(j_{1}, \ldots, j_{r}\right)=2 r-2+j_{1}+\ldots+j_{r} .
$$

Since $\operatorname{dim} H^{(r)} \times I=2 r+1$, by the multi-jet transversality theorem, we may consider the following case

$$
\begin{array}{ll}
\text { (1) } r=3, & j_{1}=j_{2}=j_{3}=1 . \\
\text { (2) } r=2, & \begin{cases}j_{1}=1, & j_{2}=2 \\
j_{1}=2, & j_{2}=1 \\
j_{1}=1, & j_{2}=1\end{cases}
\end{array}
$$

For each case, we also consider the $\mathcal{A}_{r, 1}$-orbit through each germs in the case (1) or (2). We also apply multi-transversality theorem for these orbits, we have the result by exactly the same way as the proof of Theorem 3.1, so we omit the details.

Acknowledgments This work was done during the author's stay at Hokkaido University. The author would like to thank Professor S. Izumiya very much for his help and guidance. The author would like to thank Professor G. Ishikawa for the helpful conversation. The author also want to thank Professors T. Suwa, I. Nakai, Dr. Y. Kurokawa, T. Tsukada and Department of Mathematics, Faculty of Science, Hokkaido University for their kind hospitality. Finally, the author would like to especially thank Professor T. Fukuda for his encouragement and support.

\section{References}

[1] Damon J., The unfolding and determinacy theorems for subgroups of $\mathcal{A}$ and $\mathcal{K}$. Memoirs of Amer. Math (1984), 50-306.

[2] Gibson C.G., Singular Points of Smooth Mappings. Pitman, London (1979).

[3] Golubitsky M. and Guillemin V., Stable mappings and their singularities. Graduate Texts in Math. 14 (1973), Springer-Verlag.

[4] Henry J.-P. and Merle M., Shade, Shadow and Shape. in Computational algebraic geometry (F. Eyssette and A. Galligo ed), Progress in Mathematics, Birkhäuser 109 (1993), 105-128.

[5] Ishikawa G., Izumiya S. and Watanabe K., Vector fields near a genericsubmanifolds. Geometriae Dedicata 48 (1993), 127-137.

[6] Izumiya S., Generic bifurcations of varieties. Manuscripta Math. 46 (1984), 137-164.

[7] Izumiya S. and Kossioris G.T., Geomitric singularities for solutions of single conservation laws (to appear). 
[8] Lions P.L., Rouy E. and Tourin A., Shape-from-Shading, viscosity solutions and edges. Numer. Math. 64 (1993), 323-353.

[9] Martinet J., Singularities of smooth function and maps. London Mathematical Society Lecture Note Series 58, Cambridge Univ. Press (1982).

[10] Noguchi H. and Fukuda T., Elementary Catastorophe. Kyolitsu zensho, Tokyo 208 (1976) (in Japanese).

[11] Rieger J.H., Families of maps from the plane to the plane. London Math. Soc (2) 36 (1987), 351-669.

[12] Watanabe K., Master thesis. Hokkaido University (in Japanese), (1991).

[13] Zakalyukin V.M., Reconstructions of fronts and caustics depending on a parameter and versality of mappings. Soviet Math. 27 (1983), 2713-2735.

Department of Mathematics

North East Normal University

Chang Chun 130024

P.R. China

E-mail: sun@math.hokudai.ac.jp 